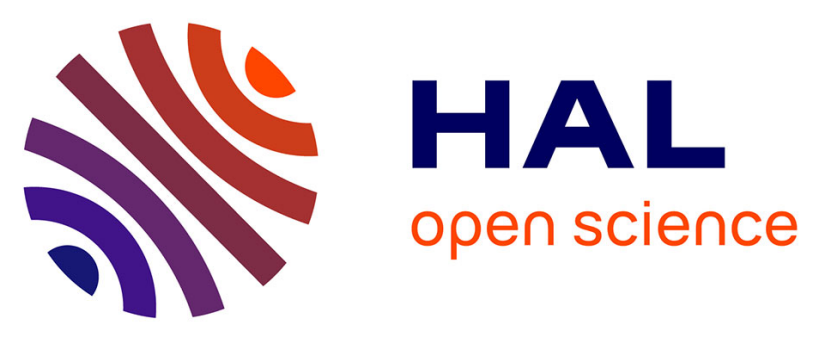

\title{
Evaluating the landscape of gene cooperativity with receptor tyrosine kinases in liver tumorigenesis using transposon-mediated mutagenesis
}

Yannan Fan, Sehrish K Bazai, Fabrice Daian, Maria Arechederra, Sylvie Richelme, Nuri Temiz, Annie Yim, Bianca Habermann, Rosanna Dono, David Largaespada, et al.

\section{To cite this version:}

Yannan Fan, Sehrish K Bazai, Fabrice Daian, Maria Arechederra, Sylvie Richelme, et al.. Evaluating the landscape of gene cooperativity with receptor tyrosine kinases in liver tumorigenesis using transposon-mediated mutagenesis. Journal of Hepatology, 2019, 70 (3), pp.470-482. 10.1016/j.jhep.2018.11.027 . hal-02368874

\section{HAL Id: hal-02368874 https://hal.science/hal-02368874}

Submitted on 22 Jan 2020

HAL is a multi-disciplinary open access archive for the deposit and dissemination of scientific research documents, whether they are published or not. The documents may come from teaching and research institutions in France or abroad, or from public or private research centers.
L'archive ouverte pluridisciplinaire HAL, est destinée au dépôt et à la diffusion de documents scientifiques de niveau recherche, publiés ou non, émanant des établissements d'enseignement et de recherche français ou étrangers, des laboratoires publics ou privés. 


\title{
Evaluating the landscape of gene cooperativity with RTKs in liver
}

\section{tumorigenesis using transposon-mediated mutagenesis}

\begin{abstract}
Yannan Fan ${ }^{1, \#}$, Sehrish K. Bazai ${ }^{1, \#}$, Fabrice Daian ${ }^{1}$, Maria Arechederra ${ }^{1}$, Sylvie Richelme ${ }^{1}$,
\end{abstract}
Nuri A. Temiz ${ }^{2}$, Annie $\mathrm{Yim}^{3}$, Bianca H. Habermann ${ }^{1}$, Rosanna Dono ${ }^{1}$, David A. Largaespada $^{2}$, and Flavio Maina ${ }^{1, *}$

${ }^{1}$ Aix Marseille Univ, CNRS, Developmental Biology Institute of Marseille (IBDM) UMR 7288, Parc Scientifique de Luminy, Marseille (France)

${ }^{2}$ Department of Pediatrics, Masonic Cancer Center, University of Minnesota, Minneapolis, MN, USA

3 Computational Biology Group, Max Planck Institute of Biochemistry, Martinsried (Germany)

${ }^{\text {\# }}$ Equal contribution

* Corresponding author

\section{Contact information of the corresponding author}

Flavio Maina

IBDM (Developmental Biology Institute of Marseille)

163 Avenue de Luminy, case 907 - 13009 Marseille (France)

Tél: +33(0)491269769 


\section{flavio.maina@univ-amu.fr}

\section{Keywords}

Liver cancer mouse model; RTKs; tumour suppressors; oncogenes; cooperative genes with RTKs; signalling; hepatocellular carcinoma; HCC patients; functional screening

\section{Electronic word count: 7469}

\section{Number of figures: 8}

\section{Conflict of interest}

The authors declare no conflicts of interest that pertain to this work.

\section{Financial support}

This work was funded by INCa (Institut National du Cancer; PL06_078 and PLBIO12-057), FdF (Fondation de France; 2014_00051580 and 2016_00067080), ARC (Association pour la Recherche contre le Cancer; SFE2011_1203807), and GEFLUC - Les Entreprises contre le Cancer to F.M. Y.F. was supported by the China Scholarship Council (201206350070). S.K.B. was supported by the Higher Education Commission (HEC) of Pakistan - France Campus. M.A. was supported by a FdF fellowship. D.A.L. is supported by an American Cancer Society Research Professorship. AY was supported by grant HA 6905/2-1 of the Deutsche Forschungsgemeinschaft (DFG) and an ERASMUS+ fellowship. The contribution of the Region Provence Alpes Côtes d'Azur and of the Aix-Marseille Université to the IBDM animal facility is also acknowledged. The funders had no role in study design, data collection and analysis, decision to publish, or preparation of the manuscript. 


\section{Authors' contributions}

Y.F.: performed the whole transposon screen and the majority of the experiments; data analysis; provided inputs on the manuscript.

S.K.B.: performed the molecular and functional validation of selected candidates.

F.D.: performed the majority of the computational work, data analysis, and interpretation.

M.A.: performed computational work with human $\mathrm{HCC}$ databases and interpretation; provided inputs on studies and on the manuscript.

S.R.: generated and characterised immorto-WT and immorto- $R 26^{M e t}$ hepatocytes; contributed to functional validation studies of candidate genes.

N.A.T.: performed transposon integration data analysis.

A.Y.: performed DAVID and KEGG analyses and contributed to computational work using the human HCC cohort from TCGA database.

B.H.H.: supervised analyses on DAVID, KEGG, and extraction of human HCC cohort data; provided inputs on the manuscript.

R.D.: contributed to establishing the $A l b-R 26^{M e t}$ mouse model; provided inputs on studies and on the manuscript.

D.A.L.: provided support for transposon sequencing and computational studies; provided input on writing the paper.

F.M.: designed the study, contributed to experimental work, analysed and interpreted data, ensured financial support, and wrote the paper. 


\begin{abstract}
Background \& Aims: The variety of alterations found in hepatocellular carcinoma (HCC) challenges the identification of functionally relevant genes and their combinatorial actions in tumorigenesis. Deregulation of Receptor Tyrosine Kinases (RTKs) is frequent in HCC, yet little is known about the molecular events that cooperate with RTKs and whether these cooperative events play an active role at the root of liver tumorigenesis.
\end{abstract}

Methods: A forward genetic screen was performed using Sleeping Beauty transposon insertional mutagenesis to accelerate liver tumour formation in a genetic context in which subtly increased MET RTK levels predispose towards tumorigenesis. Systematic sequencing of tumours identified common transposon insertion sites, thus uncovering putative RTK cooperators for liver cancer. Bioinformatic analyses were applied to transposon outcomes and human HCC datasets. In vitro and in vivo (through xenografts) functional screens were performed to assess the relevance of distinct cooperative modes to confer tumorigenic properties with RTKs.

Results: We identified 275 genes, most of which are altered in HCC patients. Unexpectedly, these genes are not restricted to a small set of pathway/cellular processes, but cover a large spectrum of cellular functions, including signalling, metabolism, chromatin remodelling, mRNA degradation, proteasome, ubiquitination, cell cycle regulation, and chromatid segregation. We validated 15 tumour suppressor candidates, as shRNA-mediated targeting confers tumorigenicity to RTK-sensitized cells, but not to cells with basal RTK levels. This demonstrates that the context of enhanced RTK levels is essential for their action in tumour initiation. 
Conclusion: Our study identifies unanticipated genetic interactions underlying gene cooperativity with RTKs in HCC. Moreover, these results show how subtly increased levels of wild-type RTKs provide a permissive context allowing a large spectrum of deregulated mechanisms to initiate liver cancer.

\section{Lay summary:}

Receptor tyrosine kinases (RTKs) are among signals frequently deregulated in hepatocellular carcinoma patients and their deregulation confers essential biological properties to cancer cells. We have applied a genetic method to randomly mutate large numbers of genes in the context of a mouse model with increased RTK levels, predisposed to develop liver cancer. We identified mechanisms that accelerate tumour formation in cooperation with enhanced RTK levels. The wide array of cellular functions among these cooperators illustrates an extraordinary capability of RTKs to render the liver more vulnerable to additional alterations, by priming cells for tumour initiation.

\section{Electronic word count of the abstract: 274}

\section{Highlights}

- A transposon genetic screen uncovered 275 putative RTK cooperators for liver cancer

- Most identified genes are also altered in HCC patients

- RTK cooperators are regulators of a large spectrum of cellular functions

- Enhanced RTK levels allow a broad range of mechanisms to initiate liver cancer 


\section{Introduction}

Hepatocellular carcinoma ( $\mathrm{HCC})$ is among the most aggressive cancers, with an increasing incidence, and few therapeutic options[1]. The exceptional investments on -omics studies over the last decade have unveiled not only an impressive list of alterations, but also a high degree of molecular heterogeneity between HCC patients[2, 3]. The uniqueness of HCC in its alterations and heterogeneity may explain how treatments effective in other cancers have largely failed when applied to $\mathrm{HCC}[4]$. Such context challenges the interpretation of -omics data, with the necessity to: 1) determine which of these alterations are functionally relevant for tumorigenic properties, 2) distinguish sets of alterations with a tumour-boosting efficiency linked to specific patient subtypes or genetic contexts, and 3) elucidate how different combinatorial alterations can lead to equivalent versus divergent fitness outcomes in cancer cells. The identification of functionally relevant signals, and of functional synergistic interactions between co-occurring events, is further complicated by the fact that some signals, although rarely mutated in HCC, are frequently activated in a high proportion of patients and are considered key regulators of tumorigenesis. It is the case, for example, for some receptor tyrosine kinase (RTK) pathway genes[4, 5]. The implication of deregulated RTK signalling in HCC is well established and RTK targeting agents are actively explored for combined therapies[6].

The Hepatocyte Growth Factor (HGF) receptor MET is one of the RTKs highly relevant in HCC. Although MET mutations are rare in HCC[7], MET is activated in close to $50 \%$ of cases[8] and this correlates with poor prognosis[9-12]. Overall, evidences implicating MET in HCC are sufficiently strong to have warranted several clinical trials of MET inhibitors[11, 13, 14 ]. We have engineered a unique conditional transgenic mouse model (R26 $6^{\text {stopMet }}$ mice)[1517] in which expression of wild-type MET can be slightly enhanced above its endogenous level in the liver $\left(A l b-R 26^{\text {Met }}\right.$ mice). We demonstrated that an approximatively 3-fold 
enhancement of MET levels in Alb-R26 $6^{\text {Met }}$ mice is sufficient to perturb tissue homeostasis The clinical relevance of this liver cancer model is supported by studies showing that Alb$R 26^{\text {Met }}$ HCCs: 1) express MET levels comparable to those reported in $\sim 20 \%$ of human HCC cases[18]; 2) exhibit active, phosphorylated MET, as observed in close to $50 \%$ of HCC patients[8]; 3) model the so-called HCC "proliferative-progenitor" patient subclass[18]; 4) while resistant to treatment with Sorafenib, are sensitive to new synthetic lethal interactions we identified[18].We recently illustrated that $A l b-R 26^{M e t} \mathrm{HCC}$ are strikingly enriched in genes that are simultaneously overexpressed and hypermethylated in gene body CpG islands (CGIs), again similar to the "proliferative-progenitor" patient subclass[19]. Such events are predictive of elevated levels of proto-oncogenes, which together act as an "oncogene module"[19]. Therefore, the $A l b-R 26^{\text {Met }}$ mouse model is particularly suitable to identify non-predictable genetic interactions underlying gene cooperativity with RTK during liver tumour initiation.

In the present study, we combined the Sleeping Beauty (SB) transposon mutagenesis system[20] with $A l b-R 26^{M e t}$ livers, as an approach to accelerate liver tumour formation. Upon characterization of transposon-insertion sites in the resulting tumours, we report the identification of 275 genes as putative cooperators of RTKs in tumorigenesis and show that most of them are altered in HCC patients. We identified co-occurring gene alteration events that are also present in HCC patients. Bioinformatic analyses illustrate that the $A l b-R 26^{M e t}$ transposon genes exert distinct functions in cells. We established the tumour-suppressive capability of 15 candidates belonging to distinct cellular mechanisms. We demonstrated that the context of enhanced RTK levels is essential, as downregulation of these tumour suppressors in wild-type cells without enhanced RTK levels does not confer tumorigenicity. Collectively, our results show how subtly increased levels in wild-type RTKs provides a permissive context allowing multiple deregulated mechanisms to initiate liver cancer. 


\section{Materials and Methods}

More detailed procedures can be found in the Supplementary Data and Supplementary CTAT Table.

\section{Genomic DNA processing, pyrosequencing, and transposon integration data analyses}

Ligation-mediated PCR with genomic DNA from 248 tumours, pyrosequencing data and findings processing was performed as previously reported[21].

\section{Co-occurring events in $A l b-$ R2 $^{M e t}$-transposon screen and in HCC patients}

Co-occurrence analysis was performed to identify genes frequently mutated in the $A l b-R 26^{M e t}$ transposon screen at a higher frequency than expected by chance[22]. A co-occurrence tumour mutation contingency table was built to calculate co-occurrence p-values by performing a Fisher exact test (FET) on each couple of $A l b-R 26^{\text {Met }}$ genes. We applied a Benjamini-Hochberg FDR correction and a threshold of significance was established at $<0.05$, leading to the identification of 119 pairs of co-occurring events (Table.S5). Several genes were identified as co-occurrences also in HCC patients based on co-concurrent differential expression $\left(\log _{2}\right.$ fold-change $>1$ or $\left.<-1\right)$. We then built a contingency table, performed FET on each gene couple, then applied a Benjamini-Hochberg FRD correction using $<0.05$ as significant level (Table.S5).

\section{Western blots}

Protein extracts from livers, tumours, immorto-WT and immorto-R26 $6^{\text {Met }}$ hepatocytes were processed for western blots, as previously described[8, 16, 23]. 


\section{Cell cultures}

1

2

Primary culture of E15.5 wild-type and $R 26^{M e t}$ embryonic hepatocytes were established as previously described[24-26]. Hepatocytes were immortalized by adapting a procedure previously reported[27]. 


\section{Results}

\section{SB-induced mutagenesis accelerates tumour development triggered by subtle enhancement of wild-type RTK MET levels.}

To search for genes whose mutations may accelerate tumorigenicity by slightly enhanced wild-type RTK MET levels[18], we combined the Alb-R26 ${ }^{\text {Met }}$ mice with the SB transposon mutagenesis system in which conditional expression of the SB transposase leads to transposon (T2/onc2) mobilization in the liver (Fig.1A, Fig.S1A,B). As the SB has the tendency to favour transposon jumping adjacent to the donor concatemers, a phenomenon known as "local hopping", we performed our screen using T2/onc2-6070 and T2/onc2-6113 lines, which carry transposon concatemers on chromosome 4 and 1, respectively. The resulting $A l b^{c r e}-T 2 / o n c 2$ $R 26^{S B /+}$ mice are referred to as "Triple ${ }^{\text {tg", }}$ (Fig.1A). We confirmed that Alb-cre-mediated excision of the stop cassette in the liver simultaneously leads to conditional expression of $\mathrm{MET}^{\mathrm{tg}}$ and SB transposase in $A l b^{\text {cre }}-T 2 / o n c 2-R 26^{S B / M e t}$ livers (referred to as "Quadruple ${ }^{\text {tg" }}$; Fig.1A-C). Quadruple ${ }^{\text {tg }}$ started to develop tumours around 30 weeks of age, a stage when tumours are still not detected in the $A l b-R 26^{\text {Met }}$ genetic setting as tumorigenesis starts around 40-48 weeks[18] (Quadruple ${ }^{\text {tg }}$ with T2/onc2-6070: 51\%; Quadruple ${ }^{\text {tg }}$ with T2/onc2-6113: 64\%; Fig.1D-F, Table.S1). In contrast, only 1 Triple $^{\text {tg }}$ in each T2/onc2 line developed tumours (Fig.1D-F, Fig.S1C Table.S1). Thus, irrespective of the initial location of the transposon concatemers, such ideal context in which only Quadruple ${ }^{\text {tg }}$ develop tumours at 30 weeks of age offered the possibility to unambiguously search for alterations underlying putative genetic interactions with wild-type RTKs during tumour initiation.

Identification of putative cancer-related genes accelerating tumorigenesis by slightly enhanced RTK MET levels. 
A total of 248 liver tumours were collected from Quadruple ${ }^{t g}$ (with either T2/onc2-6070 or

T2/onc2-6113 line) and successfully processed for pyrosequencing analysis. The procedure involved DNA barcoding of each tumour to pool and process them simultaneously, as previously reported[21]. TAPDANCE analysis identified $\sim 47,000$ non-redundant regions of transposon insertion, and defined 312 common insertion sites (CISs)[28] in tumour samples from Quadruple ${ }^{\text {tg }}$. We then annotated these CISs to the nearest mouse gene, thereby identifying 275 genes (named as Alb-R26 $6^{\text {Met }}$-transposon genes; defined by the identification of at least 8 insertions in at least 8 libraries; Fig.S1D). Based on sequencing read counts for each Alb-R26 $6^{\text {Met }}$-transposon gene, transposon insertions occurred in more than $10 \%$ of tumours for 32 genes, suggesting that these genes are among the most frequent regulators of liver tumour initiation (Fig.S1D,E, Fig.S2, Table.S2). We positioned the orientation of transposon integration in each gene and examined orientation biases of SB insertions to predict effects on genes (Fig.S1F, Table.S2). By integrating these data with the location and the distribution of transposon insertions within the gene locus, we predicted putative effects on the Alb-R26 $6^{M e t}$-transposon genes. For 103 genes, insertions were randomly distributed through the gene and occurred in both orientations or with a bias toward antisense, indicating that the contribution of these genes to tumorigenesis likely resulted from loss of gene function. These genes were thus predicted as putative tumour suppressors. For 29 genes, transposon insertions were most likely compatible with gain-of-function expression of full length or truncated forms. These genes were thus predicted as putative oncogenes. For the remaining 143 genes, orientation and position of transposon insertions could predict either gene disruption or generation of truncated forms.

Most of the putative cancer-related genes identified in the $A l b-R 2 \sigma^{M e t}$ genetic setting are deregulated in a proportion of HCC patients. 
We next determined the relevance of $A l b-R 26^{\text {Met }}$-transposon genes to human cancer and particularly to HCC pathogenesis. For comparisons between mouse and human data, the corresponding human orthologues to the Alb-R26 $6^{\text {Met }}$-transposon genes was determined. Accordingly, 267 mouse genes were matched to the corresponding human gene $(99 / 103$ predicted tumour suppressors, 29/29 predicted oncogenes, 139/143 genes for which the effect of transposon insertions was uncertain; Fig.S3A, Table.S3). By querying the Catalogue of Somatic Mutations in Cancer (COSMIC) databases, we identified 41 genes frequently mutated in human cancer $(\mathrm{P}=1.80 \mathrm{E}-20,719$ Census genes, hypergeometric-test; Fig.2A, Table.S3).

Next, we explored how alterations of Alb-R26 $6^{\text {Met }}$-transposon genes are associated with HCC pathogenesis using The Cancer Genome Atlas (TCGA) database. We analysed both expression changes (data available for 371 patients) and the presence of mutations (data available for 363 patients). Concerning mutations, we found that some of the $A l b-R 26^{M e t}$ transposon genes are also mutated in HCC patients[3] (Fig.2B, Table.S3). Overall, 71 (26\%) of Alb-R26 $6^{\text {Met }}$-transposon genes are mutated in at least $1 \%$ of patients and $218(79 \%)$ genes in at least $1 \mathrm{HCC}$ patient (Table.S3). Concerning changes in expression levels, data were available for 251 out of the 267 orthologues human genes (Fig.S3A, Table.S3). All the 251 $A l b-R 26^{M e t}$-transposon human orthologue genes are differentially expressed in at least a proportion of the 371 analysed HCC patients (Table.S3). In particular, the 29 predicted oncogenes are upregulated in a proportion of $\mathrm{HCC}$ patients ranging from $6 \%$ to $65 \%$ (Fig. $2 \mathrm{C}$, Table.S3). Concerning the 86 predicted tumour suppressors, a large set of HCC patients has consistent decreased expression levels (Fig.2D, Table.S3). In particular, 66 predicted tumour suppressors are downregulated in at least $6 \%$ of human HCC, ranging up to $81 \%$ for $G H R$ (Fig.2D). We also analysed the expression levels for the $136 \mathrm{Alb}-\mathrm{R} 26^{\mathrm{Met}}$-transposon genes for which we could not predict whether they act as tumour suppressors or oncogenes in the Alb- 
$R 26^{\text {Met }}$ genetic setting. Remarkably, a large set of human HCC is characterized by deregulation of these genes, with several of them predominantly either upregulated or downregulated (Fig.2E, Fig.S3B, Table.S3).

We then explored whether altered expression levels of the Alb-R26 ${ }^{\text {Met }}$-transposon genes correlate with MET levels in HCC patients. Concerning predicted oncogenes, there is a striking positive correlation between their overexpression and high $M E T$ expression levels (Fig.3A,B, Table.S3). For predicted tumour suppressors, we found instead that their downregulation predominantly occurred in HCC patients with low MET levels (Fig.3A,C, Table.S3). The same types of correlation characterise those genes for which the effect of transposon insertions was uncertain: whereas a large proportion of them are predominantly overexpressed in HCC patients with high $M E T$ levels, the others are mainly downregulated in patients with low MET levels (Fig.S3, Table.S3). Thus, although our screen in mice shows that MET can trigger tumorigenesis in cooperation with targeted tumour suppressors, in HCC patients with enhanced MET levels, tumorigenesis can occur even without major deregulation of this tumour suppressor set. It is tempting to speculate that MET might alleviate the need of lowering tumour suppressor levels, for example by increasing oncogene levels. Furthermore, the intriguing correlation between high levels of predicted oncogenes and MET in HCC patients is reminiscent of a novel mechanism we recently discovered, in which MET-driven HCC is characterised by high dosage of oncogenes through CGI hypermethylation in gene body[19].

Recent studies using human HCC databases have identified 5 major signalling pathways commonly altered in human HCC: PI3K/Ras signalling (here we named as RTK signalling); WNT/ $\beta$-catenin, chromatin remodelling, p53/cell cycle, oxidative/endoplasmic reticulum stress[29, 30]. To corroborate the relevance of $A l b-R 26^{M e t}$-transposon genes in HCC biology, we analysed which of them belong to these 5 major signalling pathways and identified 96 
genes. Intriguingly, the list of Alb-R26 $6^{M e t}$-transposon genes is enriched in those involved in RTK signalling (48 genes), WNT/ $\beta$-catenin (27 genes), chromatin remodelling (20 genes), rather than in p53/cell cycle (10 genes) and oxidative/endoplasmic reticulum stress ( 3 genes; Fig.2F, Table.S4). Consistently, we found activation of RTK and WNT signalling in a set of transposon tumours carrying insertions in genes acting as regulators of these pathways (Fig.S5). Thus, tumorigenesis modelled by our transposon strategy appears to occur through genetic alterations of pathways frequently perturbed in human HCC.

\section{Co-occurring events discovered in the $A l b-R 26^{M e t}$ genetic setting arise in a proportion of HCC patients.}

We searched for genes co-mutated at a frequency higher than expected by random events and identified 119 events of co-occurrence (Fig.4, Table.S5). By analysing the frequency linking each of these genes, we reconstituted nodes of interactions, identifying 4 co-occurring subnetworks of associated commonly occurrences (Fig.4, Table.S5). Beside these, 11 cooccurrences not related to the 4 subnetworks were identified (Fig.4). Strikingly, the largest cooccurring subnetwork involves 43 genes, among which the genes are involved in one or multiple types of co-occurrences (for a total of 62 co-occurrences; Fig.4, Table.S5). This large subnetwork comprises genes belonging to several pathways, including RTK signalling, chromatin remodelling, transcriptional regulation, WNT/ $\beta$-catenin, ubiquitination.

Intrigued by these findings, we asked whether co-occurring events identified in the Alb-R26 $6^{M e t}$ genetic setting could be recognized as well in HCC patients. Remarkably, 51 co-occurrences (43\%) were identified in at least $10 \%$ of HCC patients (Fig.4, Table.S5). These cooccurrences included those belonging to the large co-occurring network as well all those composing one of the three subnetworks we identified. Thus, a SB transposon mutagenesis screen in the $A l b-R 26^{M e t}$ liver cancer mouse model identifies transcriptomic and genetic 
alterations as well as co-occurring events in HCC patients. The co-occurrences may reflect possible synergistic effects between genes, increasing the likelihood that each of them ensures the tumorigenic process.

\section{The $A l b-R_{26}{ }^{M e t}$-transposon genes identify multiple enriched pathways with distinct functions in cells.}

We carried out an analysis of the Alb-R26 $6^{\text {Met }}$-transposon genes using ReactomePA databases and found that several cancer-related pathways are significantly enriched, such as WNT, B Cell Receptor, RTK signalling (Fig.S6, Table.S6). To define putative biological functions of $A l b-R 26^{M e t}$-transposon genes, we applied Database for Annotation, Visualisation, and Integrated Discovery (DAVID). These analyses allowed us to evidence an enrichment in genes related to ubiquitination, signalling pathway, metabolism, transcription, cell motility, and cancer (Table.S6). To get further insights into which molecular and cellular functions the $A l b-R 26^{M e t}$-transposon genes belong to, we performed additional enrichment analyses by applying the Enrichr tool. Concerning signalling pathways, we found an enrichment of the RTK signalling core pathway (e.g. Focal Adhesion-PI3K-mTOR, RTK effectors), TGF $\beta$, WNT, NF-kB, immune signalling (e.g. IL-6, IL-7), Delta-Notch, histone modification, metabolism, RNA processing, proteasome degradation, ubiquitination (Fig.5A, Table.S6). The Alb-R26 $6^{M e t}$-transposon genes also identify kinases based on their phosphorylation targets or the set of genes they are coexpressed with (Fig.5B, Table.S6). Similarly, the Alb-R26 ${ }^{M e t}$ transposon genes also identify a number of kinases recognizable by signatures of gene perturbations (upregulation/downregulation) following kinase knock-down (Fig.5C, Table.S6). Concerning transcription, several transcription factors were enriched for either cooccurring or interacting with sets of Alb-R26 $6^{\text {Met }}$-transposon genes (Fig.5D, Table.S6). Concerning human disease, most enriched diseases are different types of cancer (with liver 
cancer ranked third), fatty liver disease, hyperinsulinism, and hyperglycemia (Fig.5E,

Table.S6). Several Alb-R26 $6^{\text {Met }}$-transposon genes are computational predicted target of microRNA (Table.S6). Next, we searched for associations between the Alb-R2 $6^{\mathrm{Met}}$-transposon genes using STRING, which represents interactions (known or predicted) between genes (265/275 genes were found in the STRING database). Remarkably, a projection of Alb$R 26^{M e t}$-transposon genes onto the STRING protein-protein interaction network revealed that most of them are part of a network of interactions (179; Fig.6, Fig.S7,S8, Table.S6). Beside this large network, four associations were also identified (1 with 3 genes, 3 with 2 genes; Fig.6, Fig.S7,S8). These analyses illustrate how the Alb-R26 $6^{M e t}$-transposon genes are highly connected. Deregulations of these genes may propagate throughout the network, thus perturbing multiple processes. Collectively, the SB transposon mutagenesis in the $A l b-R 26^{M e t}$ genetic setting highlighted a large spectrum of pathways as potential acting modes for tumorigenicity. Moreover, these analyses uncover a putative implication in cancer of some genes for which a biological function and/or relevance in cancer was so far largely unknown (Table.S2).

\section{Comparison to other SB-induced cancer liver models identifies a set of 'putative RTK- specific genes'.}

Next, we assessed the level of specificity in gene cooperation with enhanced MET levels by comparing the Alb-R26 ${ }^{M e t}$-transposon genes with those identified in other liver SB screens: $\left.p 53^{m u t}[21]\right), \mathrm{Myc}^{\text {over }}[31], \mathrm{HBV}^{\text {tg }}[32]$, chemically induced chronic liver injury[33], hepatic steatosis[34], epithelial-mesenchymal transition[35]. Among the 275 Alb-R2 $6^{\text {Met }}$-transposon genes, $226(82 \%)$ were found in one or more SB screen cohorts. These genes are likely to be relevant to liver cancer, with a competence to cooperate with other sensitizing mutations (Fig.7A, Table.S7). Consistently, some of them have been functionally validated for their 
participation as tumorigenic drivers in other settings. Interestingly however, 49 genes were unique to our Alb-R26 $6^{\text {Met }}$-transposon cohort (Fig.7B, Table.S7). We next assessed through bioinformatics whether these genes belong to specific molecular and biological processes by applying the Enrichr tool. Several kinases are enriched according to their co-expression with sets of 'putative RTK-specific genes' (Fig.7C, Table.S7). These 'putative RTK-specific genes' also identify several kinases recognizable by signatures of gene perturbations (upregulation/downregulation) following kinase knock-down (Fig.7D, Table.S7). Among enriched kinases, are those belonging to signalling pathways such as MAPK, Insulin, and NFkB. Concerning transcription, several transcription factors are enriched according to either co-expression or to consensus targets with sets of 'putative RTK-specific genes' (Fig.7E, Table.S7). Among the top enriched transcription factors are those related to WNT- $\beta$ catenin and stemness (e.g. CHD1, CHD2, SMAD4, FOXO1, KLF4, TCF3, SOX2). Enrichments also included methylated/unmethylated $\mathrm{CpG}$ binding-transcription factors as well as genes involved in chromatin modifications. Concerning biological processes, enrichments include post-translational modifications, ubiquitination, regulation of transcription and splicing, chromatin modification, metabolism (Fig.7F, Table.S7). Thus, the 'putative RTK-specific' $A l b-R 26^{M e t}$-transposon genes exert as well distinct functions in cells and are part of multiple regulatory networks.

\section{Functional validation of a set of tumour suppressors uncovers the promiscuous capability of RTK to cooperate with distinct genes during liver tumour initiation.}

The broad spectrum of cellular regulators highlighted through bioinformatic analysis may indicate that a context with subtly enhanced RTK levels can cooperate with alterations of different types of mechanisms for liver tumorigenesis. We therefore designed a functional validation screen aimed at assessing whether a context of enhanced wild-type MET levels 
permits altered regulators of distinct cellular mechanisms to initiate transformation. For this

screen, we selected 16 predicted tumour suppressor candidates (Fig.S9) acting as regulators of distinct functions: metabolism (Adk), cell-cycle (Cacul1), proteasome (Ddi2), mRNA degradation $(D d x 6)$, chromatin remodelling (Kansl1, Kmt2e, Ncor1), signalling (Ppp6r3, Ptprd, Sorbs2), chromatin segregation (Stag2), ubiquitination (Usp34, Usp47), transcription (Zfand3). We also selected two genes which function is largely unknown: Lrch3 and WbplL. For 11 genes, mutations and downregulation in expression levels were observed in human HCC (Table.S3).

First, we generated hepatocytes from E15.5 $R 26^{\text {Met }}$ livers (as well as from E15.5 wild-type livers as control) and immortalized them with a SV40 Large T antigen (immorto- $226^{\text {Met }}$ and immorto-WT hepatocytes; Fig.8A), a strategy employed in previous studies[31]. The capacity of both immorto-R26 $6^{\text {Met }}$ and immorto-WT hepatocytes to grow in arginine-free culture conditions confirmed their liver origin. These immorto-R26 $6^{M e t}$ hepatocytes are not tumorigenic, as they do not form tumours in immune-compromised mice and colonies in anchorage-independent growth assays (Fig.S10A,B). Thus, in this cellular setting, enhanced wild-type MET expression levels, although not sufficient on its own, may prime cells towards tumorigenesis by providing a sensitized genetic background for cooperativity with additional genetic alterations. Western blot analyses revealed an approximately 3-fold increase in MET expression levels in immorto-R2 $6^{\text {Met }}$ hepatocytes compared to immorto-WT controls (Fig.8B), as previously reported in E15.5 primary embryonic hepatocytes and in Alb-R26 ${ }^{\text {Met }} \mathrm{HCC}[16]$. Qualitative analysis revealed that in both cellular systems, phosphorylation of MET and downstream signals is conditioned by HGF stimulation (Fig.8C), coherent with results in E15.5 primary embryonic hepatocytes[16, 18].

Next, we explored functionality of the 16 tumour suppressor candidates by downregulating their mRNA expression levels in immorto-R26 $6^{\text {Met }}$ hepatocytes using the shRNA targeting 
strategy (Fig.8A). For most candidate genes, we tested 2 to 5 different shRNA targeting Table.S8). Transfected cells were then used to assess whether mRNA downregulation of candidate genes conferred in vitro and in vivo tumorigenicity to immorto-R2 $6^{M e t}$ hepatocytes, thus qualifying them as functional tumour suppressors. To assess cell tumorigenicity in vitro, we performed anchorage-independent growth assays. Results show for each tested gene, the relative increase in the capacity of immorto- $R 26^{\text {Met }}$ hepatocytes to form colonies compared to cells either non-transfected or transfected with a shRNA control sequence (Fig.8D). These results were further strengthened by xenograft studies in nude mice. For 15 of the 16 candidate genes, shRNA-mediated mRNA downregulation conferred in vivo tumorigenicity to immorto-R2 $6^{\text {Met }}$ hepatocytes (Fig.8E, Fig.S11A). Outcomes also highlighted differences in 'fitness' (tumour-size), possibly reflecting varying potential of these tumour suppressors to cooperate with RTK MET when downregulated. The only exception concerned immorto$R 26^{\text {Met }}$ hepatocytes carrying a shRNA sequence targeting Zfand3 as these cells were unable to form tumours (Fig.8E, Fig.S11A).

Finally, we asked whether these genes were true cooperators, necessitating a primed 'enhanced RTK' context, or whether they would act as tumour suppressors on their own. We therefore transfected these shRNA targeting sequences into immorto-WT hepatocytes, then performed xenografts in nude mice. Remarkably, shRNA targeting these 16 tumour suppressors failed to confer tumorigenicity to immorto-WT hepatocytes (Fig.8F). Thus, our findings support a model according to which a primed context, such as the one resulting from enhanced RTK levels, is mandatory for manifesting the tumour suppressive action of these genes following shRNA targeting. As these genes operate in divergent regulatory circuits, our findings illustrate how subtly increased levels of wild-type RTKs provide a permissive setting to a plethora of deregulated mechanisms for tumorigenesis. 


\section{Discussion}

Deregulation of RTK signalling is a frequent event in HCC pathology[6]. Here, to model such deregulation, the context of slightly enhanced MET levels has been employed to identify genetic alterations that accelerate the event of liver tumour initiation. Studies have been done at stages when tumours in $A l b-R 26^{\text {Met }}$ mice are not present yet (30 weeks old mice) in order to identify the bona fide cooperators of RTKs for liver tumour initiation. The absence of tumours at this stage in Triple $e^{t g}$ (without enhanced MET levels) allows defining the $A l b-R 26^{M e t}$ transposon genes as cooperators of RTKs rather than as drivers of tumorigenicity. This is further corroborated by our functional validation studies focusing on 15 tumour suppressor candidates, for which we showed that their downregulation confers tumorigenic properties in cells with enhanced MET levels (immorto-R26 $6^{\text {Met }}$ hepatocytes), but not in immorto-WT hepatocytes. These validation studies also exemplify an extraordinary promiscuous capacity of a RTK such as MET to cooperate with multiple genes engaged in distinct cellular functions, for liver tumorigenesis. Such large spectrum of cooperativity is further illustrated by our bioinformatic studies, which evidenced enrichments among identified cooperators of distinct kinases and transcription factors operating in different pathways, as well as enrichment of distinct functions in cells. Thus, once a critical signalling threshold is attained, RTKs such as MET are rather permissive in terms of competence to act together with divergent cooperative inputs in tumorigenesis. Our findings strengthen the role of HGF/MET in HCC and exemplify how raised MET activity could play an active role at the root of liver tumorigenesis. Notably, increased HGF levels have been reported in patients affected by chronic liver disease, also recapitulated in several animal models of chronic liver dysfunction, and MET is required during liver regeneration[36-38]. Thus, in a context of chronic liver disease, an enhanced MET input, while ensuring regeneration, may render the liver more 
vulnerable to additional signals, exposing cells to signalling thresholds competent for tumour initiation.

Having identified and functionally validated a set of Alb-R26 $6^{\text {Met }}$-transposon genes, an intriguing issue to explore in future is whether and to what extent these genes represent cooperators of the MET RTK specific to the liver context, or whether these cooperators are also operational for tumorigenesis in other tissues. Our bioinformatic studies show that the $A l b-R 26^{M e t}$-transposon genes identify different types of cancer among enriched diseases, beside liver cancer. Thus, it is likely that a set of $A l b-R 26^{M e t}$-transposon genes, when altered, could act as cooperators of RTKs in several types of cancers. The $R 26^{\text {stopMet }}$ genetic system offers the possibility of enhancing MET expression in the tissue of choice, according to the tissue-specific Cre employed. Therefore, such a genetic approach allows addressing the issue of tissue-specificity for qualitative signalling cooperation with RTKs in future. This may be particularly relevant in the subset of vulnerable tissues in which a subtle increase in wild-type MET expression levels is poorly tolerated, consequently destabilizing cell biological properties favouring a tumour initiation event[39], particularly in tissues where the MET oncogenic action is well established.

The comparison we performed between the outcomes of SB transposon mutagenesis carried out in the present $A l b-R 26^{M e t}$ genetic setting and in other settings[21, 31-35] revealed that among the $275 A l b-R 26^{M e t}$-transposon genes, aside from a large group of common hits, 49 genes were only found in the $A l b-R 26^{M e t}$ context, which we named 'putative RTK-specific genes'. For a proportion of them, it is possible that they could have been missed in previous screens because transposon mutagenesis does not reach saturation levels, particularly with small numbers of analysed tumours. Additionally, differences in mouse genetic background, SB transposon versions, sequencing and bioinformatic analyses, can also limit comparisons of genes identified through SB screens[33]. In spite of these limitations, it is tempting to 
speculate that when altered, at least some of these 'putative RTK-specific genes' may be competent to exert their tumorigenic potential predominantly in a context with enhanced RTK levels (and possibly in few other genetic settings), rather than together with any kind of liversensitizing signals. The possibility of such of genetic specificity in cooperation for tumorigenesis has been recently proposed through forward genetic screens in mice[40, 41].

Altogether, the knowledge of these Alb-R26 $6^{\text {Met }}$-transposon genes that we identified in a clinically relevant liver cancer genetic model may contribute to the identification of drivers operating in HCC patients as well as genetic interactions with altered RTKs in human HCC. Ultimately, our results will inform the design of new combinatorial therapies for HCC patient subsets. 


\section{Acknowledgements}

These results are in part based upon public data generated by TCGA Research Network: http://cancergenome.nih.gov/. We are particularly grateful to F. Helmbacher for extremely valuable feedback on the study and for help with improving the manuscript. We thank: Á. M. Martínez-Valverde for providing us the SV40 Large T Antigen to immortalize the embryonic hepatocytes; all members of our labs for helpful discussions and comments; V. Girod-David and L. Jullien for excellent help with mouse husbandry. 


\section{References}

[1] Llovet JM, Zucman-Rossi J, Pikarsky E, Sangro B, Schwartz M, Sherman M, et al. Hepatocellular carcinoma. Nat Rev Dis Primers 2016;2:16018.

[2] Zucman-Rossi J, Villanueva A, Nault JC, Llovet JM. Genetic Landscape and Biomarkers of Hepatocellular Carcinoma. Gastroenterology 2015;149:1226-1239 e1224.

[3] Cancer Genome Atlas Research Network. Electronic address wbe, Cancer Genome Atlas Research N. Comprehensive and Integrative Genomic Characterization of Hepatocellular Carcinoma. Cell 2017;169:1327-1341 e1323.

[4] Llovet JM, Villanueva A, Lachenmayer A, Finn RS. Advances in targeted therapies for hepatocellular carcinoma in the genomic era. Nat Rev Clin Oncol 2015;12:408-424.

[5] Maina F. Strategies to overcome drug resistance of receptor tyrosine kinaseaddicted cancer cells. Current medicinal chemistry 2014;21:1607-1617.

[6] Worns MA, Galle PR. HCC therapies--lessons learned. Nat Rev Gastroenterol Hepatol 2014;11:447-452.

[7] Park WS, Dong SM, Kim SY, Na EY, Shin MS, Pi JH, et al. Somatic mutations in the kinase domain of the Met/hepatocyte growth factor receptor gene in childhood hepatocellular carcinomas. Cancer Res 1999;59:307-310.

[8] Furlan A, Stagni V, Hussain A, Richelme S, Conti F, Prodosmo A, et al. Abl interconnects oncogenic Met and p53 core pathways in cancer cells. Cell Death Differ 2011;18:1608-1616.

[9] Kaposi-Novak P, Lee JS, Gomez-Quiroz L, Coulouarn C, Factor VM, Thorgeirsson SS. Met-regulated expression signature defines a subset of human hepatocellular carcinomas with poor prognosis and aggressive phenotype. J Clin Invest 2006;116:1582-1595.

[10] Goyal L, Muzumdar MD, Zhu AX. Targeting the HGF/c-MET pathway in hepatocellular carcinoma. Clin Cancer Res 2013;19:2310-2318. 
[11] Giordano S, Columbano A. Met as a therapeutic target in HCC: facts and hopes. J Hepatol 2014;60:442-452.

[12] Horwitz E, Stein I, Andreozzi M, Nemeth J, Shoham A, Pappo O, et al. Human and mouse VEGFA-amplified hepatocellular carcinomas are highly sensitive to sorafenib treatment. Cancer Discov 2014;4:730-743.

[13] Du Z, Caenepeel S, Shen Y, Rex K, Zhang Y, He Y, et al. Preclinical Evaluation of AMG 337, a Highly Selective Small Molecule MET Inhibitor, in Hepatocellular Carcinoma. Mol Cancer Ther 2016;15:1227-1237.

[14] You H, Ding W, Dang H, Jiang Y, Rountree CB. c-Met represents a potential therapeutic target for personalized treatment in hepatocellular carcinoma. Hepatology 2011;54:879-889.

[15] Genestine M, Caricati E, Fico A, Richelme S, Hassani H, Sunyach C, et al. Enhanced neuronal Met signalling levels in ALS mice delay disease onset. Cell Death Dis 2011;2:e130.

[16] Fan Y, Richelme S, Avazeri E, Audebert S, Helmbacher F, Dono R, et al. TissueSpecific Gain of RTK Signalling Uncovers Selective Cell Vulnerability during Embryogenesis. PLoS genetics 2015;11:e1005533.

[17] Tonges L, Ostendorf T, Lamballe F, Genestine M, Dono R, Koch JC, et al. Hepatocyte growth factor protects retinal ganglion cells by increasing neuronal survival and axonal regeneration in vitro and in vivo. J Neurochem 2011;117:892-903.

[18] Fan YN, Arechederra M, Richelme S, Daian F, Novello C, Calderaro J, et al. A Phosphokinome-Based Screen Uncovers New Drug Synergies for Cancer Driven by LiverSpecific Gain of Nononcogenic Receptor Tyrosine Kinases. Hepatology 2017;66:1644-1661.

[19] Arechederra M, Daian F, Yim A, Bazai SK, Richelme S, Dono R, et al. Hypermethylation of gene body $\mathrm{CpG}$ islands predicts high dosage of functional oncogenes in liver cancer. Nature Communications 2018;9. 
[20] Uren A, Berns A. Jump-starting cancer gene discovery. Nat Biotechnol 2009;27:251252.

[21] Keng VW, Villanueva A, Chiang DY, Dupuy AJ, Ryan BJ, Matise I, et al. A conditional transposon-based insertional mutagenesis screen for genes associated with mouse hepatocellular carcinoma. Nat Biotechnol 2009;27:264-274.

[22] Rahrmann EP, Watson AL, Keng VW, Choi K, Moriarity BS, Beckmann DA, et al. Forward genetic screen for malignant peripheral nerve sheath tumor formation identifies new genes and pathways driving tumorigenesis. Nat Genet 2013;45:756-766.

[23] Furlan A, Roux B, Lamballe F, Conti F, Issaly N, Daian F, et al. Combined drug action of 2-phenylimidazo[2,1-b]benzothiazole derivatives on cancer cells according to their oncogenic molecular signatures. PLoS One 2012;7:e46738.

[24] Maina F, Pante G, Helmbacher F, Andres R, Porthin A, Davies AM, et al. Coupling Met to specific pathways results in distinct developmental outcomes. Mol Cell 2001;7:12931306.

[25] Moumen A, Ieraci A, Patane S, Sole C, Comella JX, Dono R, et al. Met signals hepatocyte survival by preventing Fas-triggered FLIP degradation in a PI3k-Akt-dependent manner. Hepatology 2007;45:1210-1217.

[26] Furlan A, Lamballe F, Stagni V, Hussain A, Richelme S, Prodosmo A, et al. Met acts through Abl to regulate p53 transcriptional outcomes and cell survival in the developing liver. J Hepatol 2012;57:1292-1298.

[27] Gonzalez-Rodriguez A, Clampit JE, Escribano O, Benito M, Rondinone CM, Valverde AM. Developmental switch from prolonged insulin action to increased insulin sensitivity in protein tyrosine phosphatase 1B-deficient hepatocytes. Endocrinology 2007;148:594-608. 
[28] Sarver AL, Erdman J, Starr T, Largaespada DA, Silverstein KAT. TAPDANCE: An automated tool to identify and annotate transposon insertion CISs and associations between CISs from next generation sequence data. Bmc Bioinformatics 2012;13.

[29] Guichard C, Amaddeo G, Imbeaud S, Ladeiro Y, Pelletier L, Maad IB, et al. Integrated analysis of somatic mutations and focal copy-number changes identifies key genes and pathways in hepatocellular carcinoma. Nat Genet 2012;44:694-698.

[30] Totoki Y, Tatsuno K, Covington KR, Ueda H, Creighton CJ, Kato M, et al. Transancestry mutational landscape of hepatocellular carcinoma genomes. Nat Genet 2014;46:1267-1273.

[31] O'Donnell KA, Keng VW, York B, Reineke EL, Seo D, Fan D, et al. A Sleeping Beauty mutagenesis screen reveals a tumor suppressor role for Ncoa2/Src-2 in liver cancer. Proc Natl Acad Sci U S A 2012;109:E1377-1386.

[32] Bard-Chapeau EA, Nguyen AT, Rust AG, Sayadi A, Lee P, Chua BQ, et al. Transposon mutagenesis identifies genes driving hepatocellular carcinoma in a chronic hepatitis B mouse model. Nat Genet 2014;46:24-32.

[33] Riordan JD, Feddersen CR, Tschida BR, Beckmann PJ, Keng VW, Linden MA, et al. Chronic liver injury alters driver mutation profiles in hepatocellular carcinoma in mice. Hepatology 2017.

[34] Tschida BR, Temiz NA, Kuka TP, Lee LA, Riordan JD, Tierrablanca CA, et al. Sleeping Beauty Insertional Mutagenesis in Mice Identifies Drivers of Steatosis-Associated Hepatic Tumors. Cancer Research 2017;77:6576-6588.

[35] Kodama T, Newberg JY, Kodama M, Rangel R, Yoshihara K, Tien JC, et al. Transposon mutagenesis identifies genes and cellular processes driving epithelialmesenchymal transition in hepatocellular carcinoma. Proc Natl Acad Sci U S A 2016;113:E3384-3393. 
[36] Huh CG, Factor VM, Sanchez A, Uchida K, Conner EA, Thorgeirsson SS. Hepatocyte growth factor/c-met signaling pathway is required for efficient liver regeneration and repair. Proc Natl Acad Sci U S A 2004;101:4477-4482.

[37] Shiota G, Okano JI, Kawasaki H, Kawamoto T, Nakamura T. Serum Hepatocyte Growth-Factor Levels in Liver-Diseases - Clinical Implications. Hepatology 1995;21:106112.

[38] Borowiak M, Garratt AN, Wustefeld T, Strehle M, Trautwein C, Birchmeier C. Met provides essential signals for liver regeneration. Proc Natl Acad Sci U S A 2004;101:1060810613.

[39] Peacock JD, Pridgeon MG, Tovar EA, Essenburg CJ, Bowman M, Madaj Z, et al. Genomic Status of MET Potentiates Sensitivity to MET and MEK Inhibition in NF1-Related Malignant Peripheral Nerve Sheath Tumors. Cancer Res 2018;78:3672-3687.

[40] Rogers ZN, McFarland CD, Winters IP, Seoane JA, Brady JJ, Yoon S, et al. Mapping the in vivo fitness landscape of lung adenocarcinoma tumor suppression in mice. Nat Genet 2018;50:483-486.

[41] Wangensteen KJ, Wang YJ, Dou Z, Wang AW, Mosleh-Shirazi E, Horlbeck MA, et al. Combinatorial genetics in liver repopulation and carcinogenesis with a novel in vivo CRISPR activation platform. Hepatology 2017.

[42] Ponzetto C, Bardelli A, Zhen Z, Maina F, dalla Zonca P, Giordano S, et al. A multifunctional docking site mediates signaling and transformation by the hepatocyte growth factor/scatter factor receptor family. Cell 1994;77:261-271.

[43] Maina F, Casagranda F, Audero E, Simeone A, Comoglio P, Klein R, et al. Uncoupling of Grb2 from the Met receptor in vivo reveals complex roles in muscle development. Cell 1996;87:531-542. 


\section{Figure legends}

Fig.1. Genetic settings employed for the $A l b-R_{26}{ }^{M e t}$-transposon screen. (A) Schematic representation of the three genetic settings employed for the SB transposon mutagenesis screen. Concerning the T2/onc transgenics, a concatemer of transposons is present on chromosome 4 (6070 line) or chromosome 1 (6113 line). (B) Immunohistochemical analysis reporting $\beta$-galactosidase and SB transposase expression in the indicated genetic settings. (C) Western-blot analysis of total protein extracts from Triple ${ }^{\text {tg }}$, Quadruple $^{\text {tg }}$, and control livers. Note $\mathrm{MET}^{\mathrm{tg}}$ expression specifically in Quadruple ${ }^{\text {tg }}$ (detected by anti-human MET antibodies). Actin was used as a loading control. (D) Examples of liver tumours in Quadruple $^{\text {tg }}$ (arrows). Tumour size ranged from 1 to $4 \mathrm{~mm}$ diameters (Table.S1). Example of multiple tumours (left) or single small tumours within the liver lobes (right) are shown. (E,F) Tumour formation in Alb-R26 ${ }^{\text {Met }}$, Triple ${ }^{\text {tg }}$, Quadruple $^{\text {tg }}$ at 30 weeks of age. Data correspond to T2/onc2-6070 (E) and T2/onc2-6113 (F) lines. Each dot corresponds to a mouse. The percentage (and numbers) of mice developing liver tumours is indicated.

Fig.2. Cross-species comparison implicates the $A l b-R_{26}{ }^{M e t}$-transposon genes in HCC pathogenesis and a proportion of them in human cancers. (A) Word-cloud diagram reporting the $41 \mathrm{Alb}-\mathrm{R} 26^{\mathrm{Met}}$-transposon genes frequently mutated in human cancer according to the COSMIC Census gene databases (Table.S3). (B) Word-cloud diagram reporting the $A l b-R 26^{M e t}$-transposon genes mutated in HCC patients. The 9 genes significantly mutated in human HCC are indicated in black (p-value $<0.05$ ). The 70 genes mutated in more than $1 \%$ of HCC patients are indicated in grey (Table.S3). In A and B, the size of each gene name is representative of the percentage of patients with mutations in the corresponding genes. (C-E) RNA-seq data from human HCC patients were queried for alterations in $A l b-R 26^{M e t}$ transposon gene expression (Table.S3). (C) Scatterplot depicting HCC patients with 
upregulation $\left(\log _{2}\right.$ fold change $\left.(\mathrm{FC})>1\right)$ of predicted oncogenes according to effects of transposon insertion. (D) Scatterplot depicting HCC patients with downregulation ( $\log _{2}$ fold change $(\mathrm{FC})<1)$ of predicted tumour suppressors according to effects of transposon insertion. (E) Scatterplot depicting HCC patients with deregulation $\left(\log _{2}\right.$ fold change $(\mathrm{FC})>$ or $\left.<1\right)$ of genes for which transposon insertions did not allowed unambiguous effect prediction. Only $A l b-R 26^{\text {Met }}$-transposon genes deregulated in at least $30 \%$ of $\mathrm{HCC}$ patients are reported. The other genes are shown in Fig.S2B. (F) Alb-R26 $6^{\text {Met }}$-transposon genes belonging to the 5 major pathways reported to be altered in HCC patients[29, 30]: RTK signalling, WNT/ $\beta$-catenin, chromatin remodelling, p53/cell cycle, oxidative/endoplasmic reticulum stress. Note an enrichment in genes participating to RTK signalling, WNT/ $\beta$-catenin, and chromatin remodelling. The RTK core pathway includes genes either regulating or participating to the RTK signalling. Some positive and negative regulations are indicated. Concerning the $\mathrm{WNT} / \beta$-catenin, most genes are organized as negative or positive regulators; genes belonging to the WNT/Hippo pathway crosstalk are also highlighted. Concerning chromatin remodelling, most genes are grouped into regulators of histone methylation, histone acetylation, DNA replication. Concerning p53/cell cycle, genes are organized according to their implication in mitosis, cyclin, p53 regulators.

Fig.3. Predicted $A l b-R 26^{M e t}$-transposon oncogenes are predominantly upregulated in HCC patients with high MET levels, whereas predicted tumour suppressors are predominantly downregulated in patients with low MET. (A) Heat-map reporting expression levels of predicted oncogenes and tumour suppressors (rows) in individual HCC patients (columns; organised according to the higher number of patients with upregulated oncogenes and downregulated tumour suppressors). Red: upregulated oncogenes; green: downregulated tumour suppressors. $(\mathrm{B}, \mathrm{C})$ In the graphs, each dot corresponds to a given oncogene (B) or tumour suppressor (C), and their position to the percentage of patients in 
which the gene is upregulated or downregulated, respectively. Significant differences are indicated on the top: $* *: \mathrm{P}<0.01 ; * * *: \mathrm{P}<0.001$.

Fig.4. Co-occurring events in the $A l b-R 26^{M e t}$-transposon screen can be grouped into interacting networks, with a large proportion present in HCC patient subsets. Based on pairwise comparisons between each Alb-R2 $6^{\text {Met }}$-transposon genes and Fisher's exact test, 119 co-occurrences were identified in the $A l b-R 26^{\text {Met }}$ liver cancer model. Beside the 11 cooccurring events concerning only two genes (bottom-right), a large network (composed by 43 genes) and three subnetworks were spotted (Table.S5). Among the 4 co-occurring subnetworks, one of them includes Zfp568, Luc7l2 together with several Ugtla family members, encoded by a single gene locus (Fig.S12). 51 co-occurrences are present in at least $10 \%$ of HCC patients, shown in the figure with blue interconnecting lines in which the thickness between two genes represents the percentage of co-occurring events in HCC patients. Co-occurring alterations in at least $15 \%$ of human $\mathrm{HCC}$ are indicated in blue (Table.S5).

Fig.5. Multiple signalling and transcription networks are enriched by the $A l b-R 26^{M e t}$ transposon genes. Enrichment analyses by applying the Enrichr tool. (A) Histogram reporting the cell signalling pathway enrichment, ordered according to the combines score (top: WikiPathways database; bottom: Reactome database). (B) Histogram reporting the kinases identified based on their phosphorylation targets (top: Kinase Enrichment Analysis, KEA database) or the set of genes they are coexpressed with (bottom: Co-expressed with kinases, ARCHS4 kinases Coex database) sets of identified genes. (C) Histogram reporting kinases recognizable by signatures of gene perturbations (upregulation/downregulation) following kinase knock-down. (D) Histogram reporting transcription factors enriched for either cooccurring (left: Enrichr submission TF-Gene Co-occurrence database) or interacting (right: 
Transcription Factor PPIs database) with sets of Alb-R26 $6^{M e t}$-transposon genes. Additional analyses based on ChEA, Encode \& ChEA consensus TFs from ChIP-X, and ARCHS4 TFs coexp databases are reported in Table.S6. (E) Histogram reporting enrichment of human diseases Jensen Diseases database). In B,C,D, enriched kinases and transcription factors belonging to the Alb-R26 $6^{M e t}$-transposon genes are indicated in red. In all panels, enrichments are ordered according to combined scores (values are indicated on the $\mathrm{x}$ axis).

Fig.6. Most of the Alb-R26 ${ }^{M e t}$-transposon genes are part of networks of interactions. Projection of $A l b-R 26^{M e t}$-transposon genes onto the STRING protein-protein interaction network highlights a large network of 179 connected nodes. Associations between 3 genes (1) and 2 genes (3) are also indicated. The colour of the edges correspond to different types of interactions reported on the bottom. Nodes not connected (77) are shown in Fig.S6.

Fig.7. 'Putative RTK-specific genes' identified in the $A l b-R 26^{M e t}$ genetic setting. (A) Schematic representation reporting the genetic comparison to SB screens performed in other HCC models (steatosis, chronic injury, epithelial-mesenchymal transition (EMT), HBV, p53, MYC mutant background)[21, 31-35]. Vertical bars: number of Alb-R2 $6^{\text {Met }}$-transposon genes overlapping with other studies. Horizontal bars: total number of genes for each screen used for analysis. Dark grey squares: datasets used in each comparison. (B) List of $A l b-R 26^{M e t}$ transposon genes not identified in previous liver transposons studies, named 'putative RTKspecific genes', ordered according to the mutation frequency. (C-F) Enrichment analyses by applying the Enrichr tool. (C) Clustergram reporting the kinases enriched according to their co-expression with 'putative RTK-specific genes' (Co-expressed with kinases, ARCHS4 kinases Coex database). (D) Clustergram reporting kinases recognizable by signatures of gene perturbations (upregulation/downregulation) following kinase knock-down (LINCS L1000 Kinase perturbations up/down databases). (E) Clustergram reporting transcription factors 
enriched according to either co-occurring (left: Enrichr submission TF-Gene Co-occurrence database) or the consensus targets (right: ENCODE and ChEA consensus TFs from ChIP-X database) with sets of 'putative RTK-specific genes'. (F) Clustergram reporting enrichment of biological processes (Gene Ontology Biological processes database). In D and E, enriched kinases and transcription factors belonging to the Alb-R2 $6^{M e t}$-transposon genes are indicated in red.

Fig.8. Generation of cellular systems for functional exploration of $A l b-R 26^{M e t}$-transposon genes and functional validations in vitro and in vivo. (A) Schematic representation of the overall strategy employed for generation of immorto-R $26^{M e t}$ and immorto-WT hepatocytes and their use to assess functionality of $A l b-R 26^{M e t}$-transposon gene sets. $R 26^{M e t}$ (top) or wild-type (bottom) livers were dissected from E15.5 embryos and use to established primary embryonic hepatocytes, which were then immortalized with a retrovirus carrying the SV40 LargeT antigen. Cells were then transfected with plasmids carrying the shRNA sequence targeting the tumour suppressor candidates. Cells were used for molecular analyses to evaluate the extent of gene downregulation, and for functional studies using anchorage-independent growth (softagar) assays and xenografts in nude mice. (B) Quantification and analysis of $\mathrm{MET}^{\mathrm{tg}}$ versus endogenous MET levels using protein extracts from immorto-WT and immorto-R26 $6^{\text {Met }}$ hepatocytes. Note that the MET protein levels present in $10 \mu \mathrm{g}(\gamma)$ of total immorto-R26 $6^{\text {Met }}$ hepatocyte protein extracts are comparable to those found in $30 \mu \mathrm{g}$ of control extracts. The anti-MET ${ }^{25 \mathrm{H} 2}$ antibodies recognize both endogenous and $\mathrm{MET}^{\mathrm{tg}}$ proteins. (C) Western blot analysis of total protein extracts from immorto-WT and immorto-R2 $6^{\text {Met }}$ hepatocytes before and after HGF stimulation $(50 \mathrm{ng} / \mathrm{ml})$. Results show that in these cells phosphorylation of MET on $\operatorname{Tyr}_{1234-1235}$ (within the kinase domain reflecting MET activity), $\mathrm{Tyr}_{1003}$, (critical for MET protein ubiquitination/degradation), and $\operatorname{Tyr}_{1349}$ (one of the two multifunctional docking sites critical for MET signalling[24, 42, 43]) as well as of GAB1 on $\operatorname{Tyr}_{627}$ are is dependent on 
HGF stimulation. Basal phosphorylation levels of AKT and ERKs are increased upon HGF stimulation. A scheme summarizing the main tyrosine residues in MET is reported on the right. In B and C, Actin was used as a loading control. (D) Graph reporting the number of colonies formed in anchorage-independent growth assays by immorto-R2 $6^{\text {Met }}$ hepatocytes transfected with a shRNA sequence targeting the indicated $R 26^{M e t}$-transpson genes compared to control cells (either non-transfected or transfected with a shRNA control sequence). Note colony number formation of immorto-R2 $6^{\text {Met }}$ hepatocytes with downregulated candidate genes compared with control cells. (E) Quantification of tumour volume in mice injected subcutaneously with immorto-R2 $6^{\text {Met }}$ hepatocytes transfected with plasmid carrying the shRNA sequence targeting the indicated gene compared with control cells (either non-transfected or transfected with a control vector). The dark-dot in Adk corresponds to a tumour dissected for ethics. (F) Quantification of tumour volume in mice injected sub-cutaneously with immortoWT hepatocytes transfected with plasmid carrying the shRNA sequence targeting the indicated gene compared with control cells (either non-transfected or transfected with a control vector). Note no tumour formation. Significant differences compared to controls are indicated on the top. $*: \mathrm{P}<0.05 ; * *: \mathrm{P}<0.01 ; * * *: \mathrm{P}<0.001$ (n.d.: not determined). 

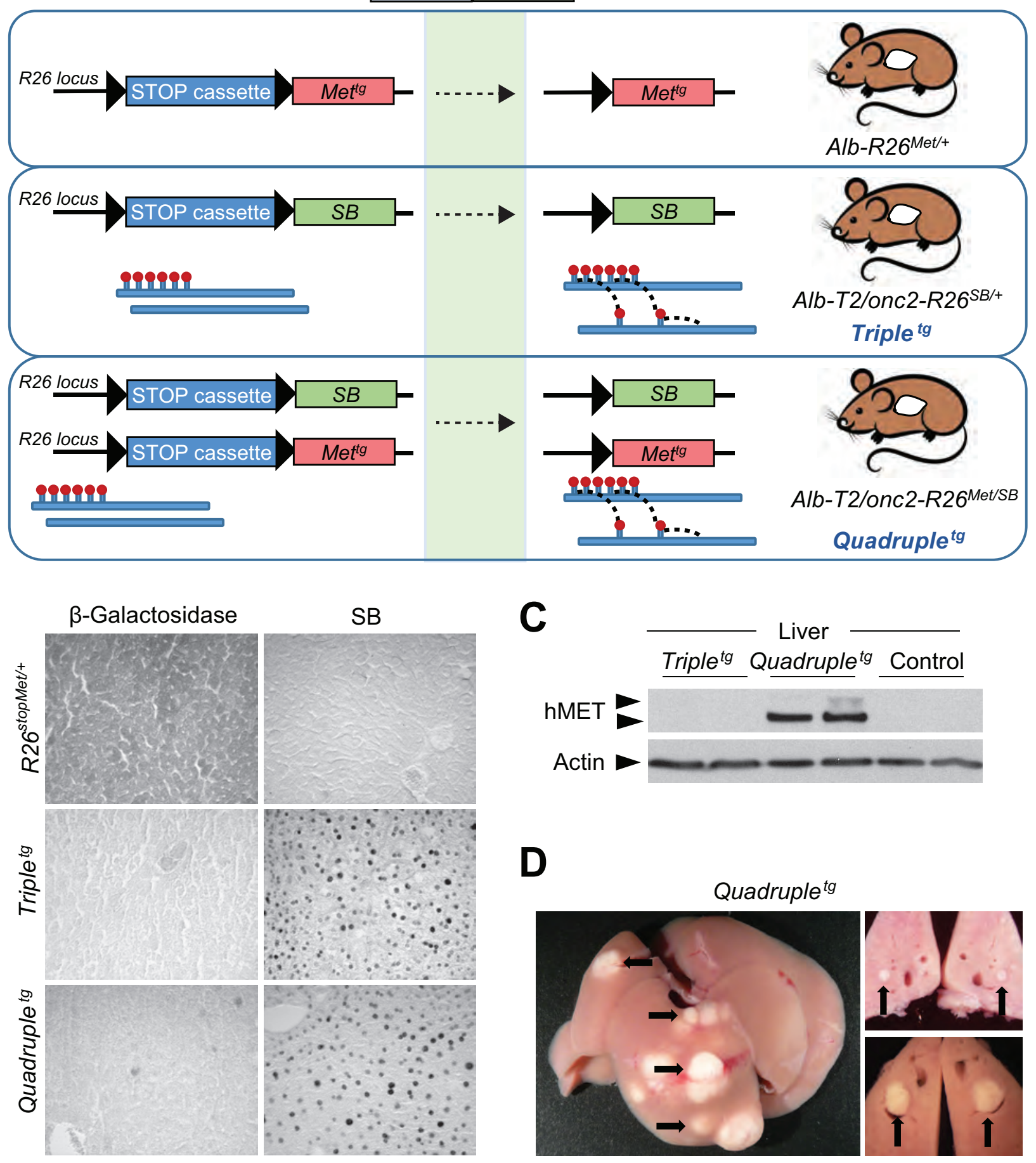

C

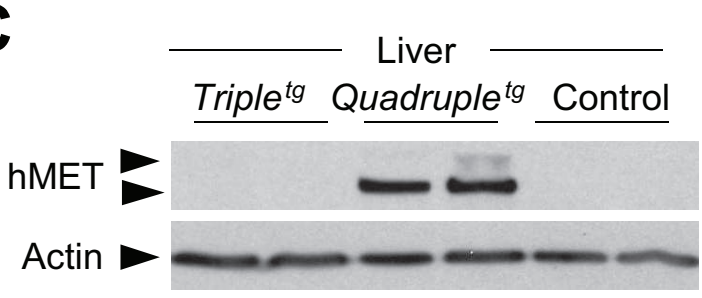

D

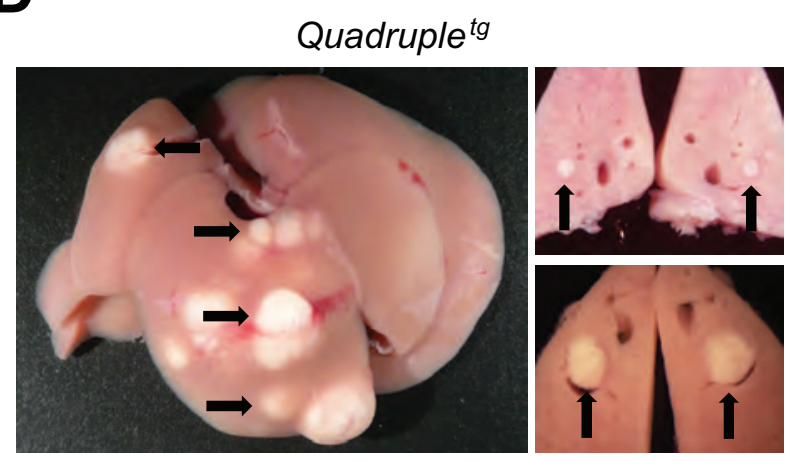

E

_ T2/onc2-6070 line (chr. 4)

$\mathbf{F}$

T2/onc2-6113 line (chr. 1) -
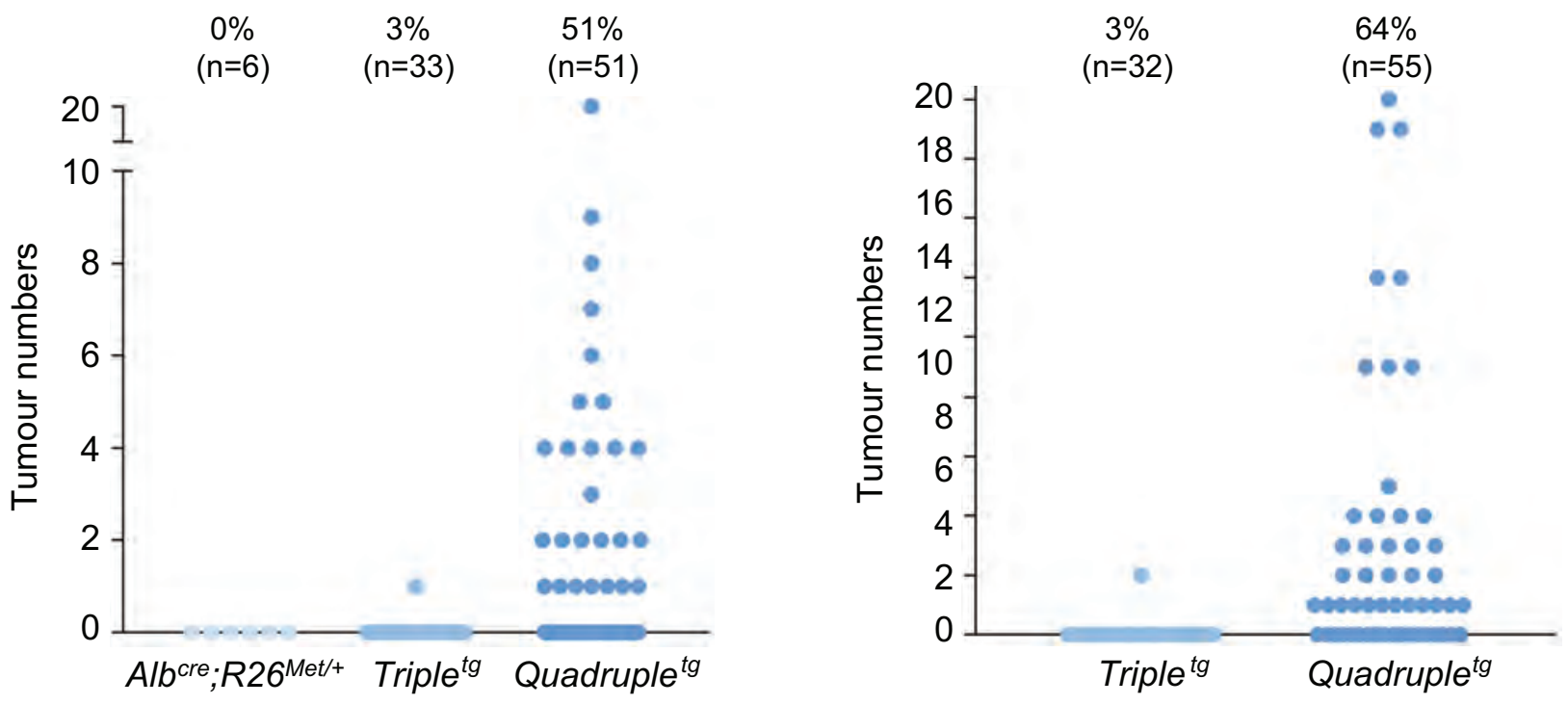

Figure 1 


\section{Figure 2}

A mutated genes in cancer patients

$\sum_{0}^{N}$ LABP4B

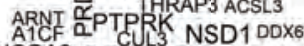
NCOA2 CREBBP CTNND1 ARIB PTPRD SF3B1 BRD4

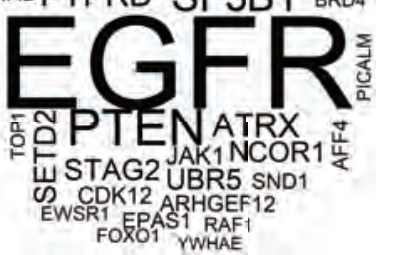

B mutated genes in HCC patients

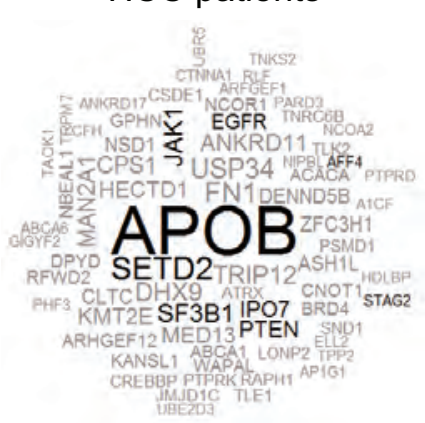

C Predicted Oncogenes

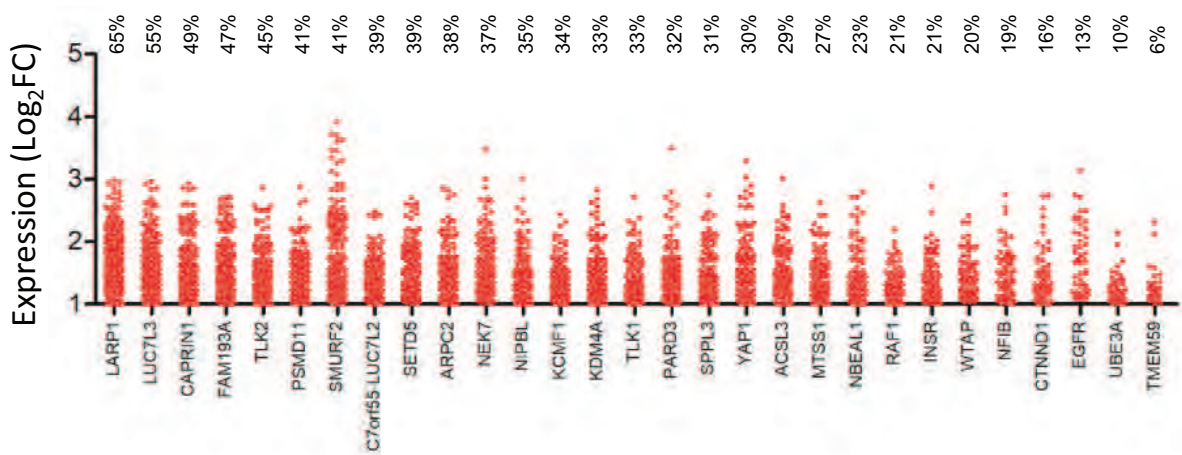

Predicted Tumour Suppressors

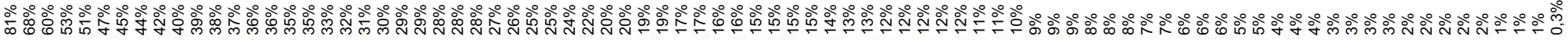

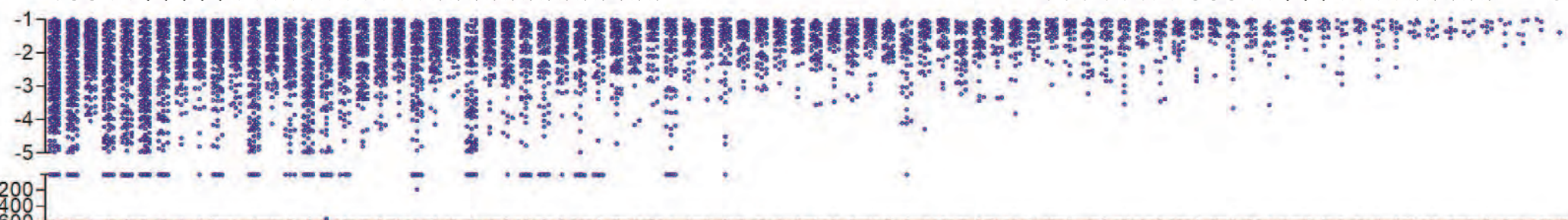

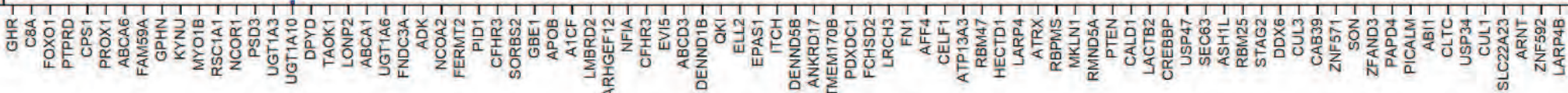

E

Deregulated genes

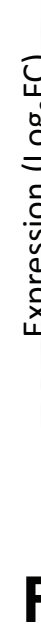

10

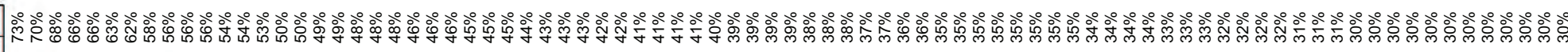
6 .

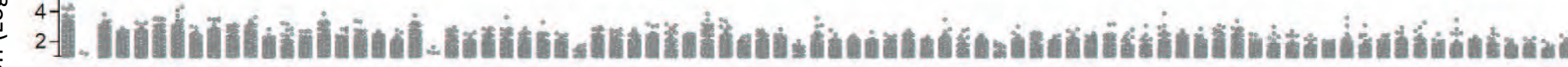

RTK signalling

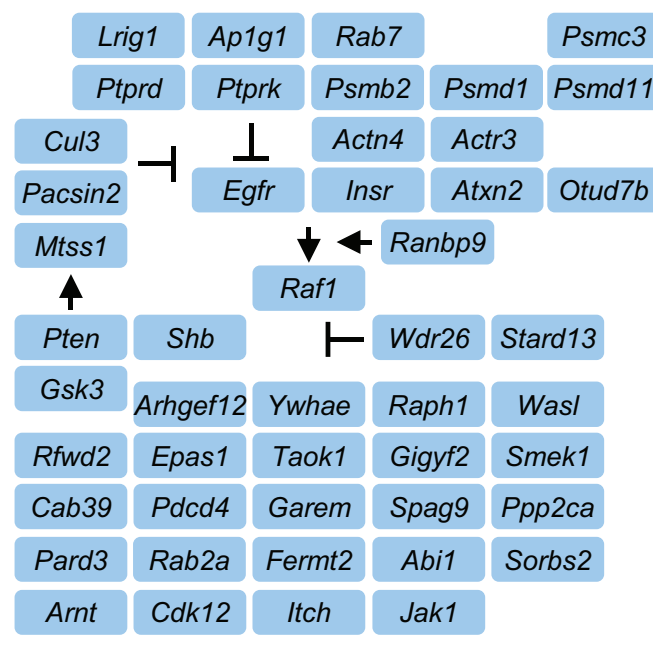

WNT/ $\beta$-catenin

\begin{tabular}{|lcc} 
negative & $\begin{array}{c}\text { positive } \\
\text { regulators } \\
\text { regulators }\end{array}$ & $\begin{array}{c}\text { Hippo } \\
\text { crosstalk }\end{array}$ \\
Gsk3 & Ctnnd1 & Yap1 \\
Ptprk & Smurf2 & Mob1b \\
Wdr26 & Tnks2 & Mob3b \\
Tle1 & Zranb1 & \\
Cul3 & Tmem59 & \\
Usp34 & Tmem170b \\
Hectd1 & & \\
Fermt2 & Foxo1 & Actn4 \\
\hline Psmb2 & Psmd1 & Psmc3 \\
\hline Numb & Cltc & Wwp11 \\
\hline
\end{tabular}

chromatin remodelling :

\begin{tabular}{|c|c|c|}
\hline $\begin{array}{l}\text { histone } \\
\text { methyl. }\end{array}$ & $\begin{array}{l}\text { histone } \\
\text { acetyl. }\end{array}$ & $\begin{array}{c}\text { DNA } \\
\text { replication }\end{array}$ \\
\hline Setd2 & Ankrd1 & Ankrd17 \\
\hline Ash1I & Setd5 & Tlk2 \\
\hline$K d m 4 a$ & Kans/1 & Atrx \\
\hline $\mathrm{Km} 2 \mathrm{e}$ & Ncor1 & Aff4 \\
\hline$N s d 1$ & & Top1 \\
\hline Prdm2 & & Tlk1 \\
\hline \multicolumn{3}{|l|}{ Jmjd1c } \\
\hline \multicolumn{3}{|r|}{ Uhrf2 } \\
\hline & $\mathrm{rd} 4$ & Dcaf8 \\
\hline
\end{tabular}

p53/cell cycle

\begin{tabular}{|l|l|l|} 
mitosis & \multicolumn{1}{c}{ cyclin } \\
regulators & \multicolumn{1}{c}{ regulators } \\
Stag2 & Ccny & Rfwd2 \\
\hline Nek7 & Evi5 & \\
\hline Atrx & Ppm1b & \\
& Cacul1 \\
& Ccnt2 \\
\hline & Caprin1 \\
\hline
\end{tabular}

oxidative/ER stress

Txndc11

Mtfr1 
Sigure 3

HCC patients with

HCC patients with

upregulated MET

downregulated MET
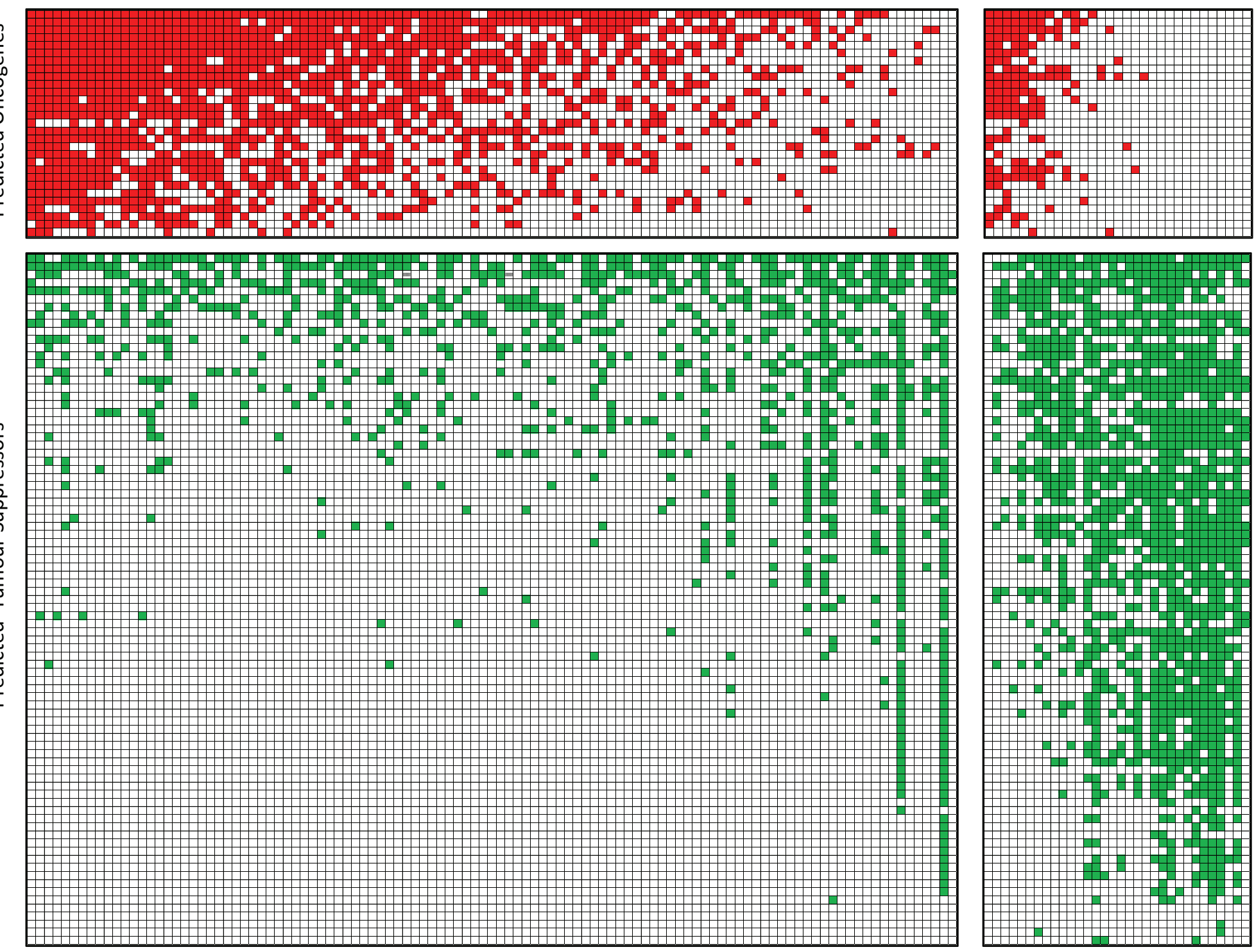

B

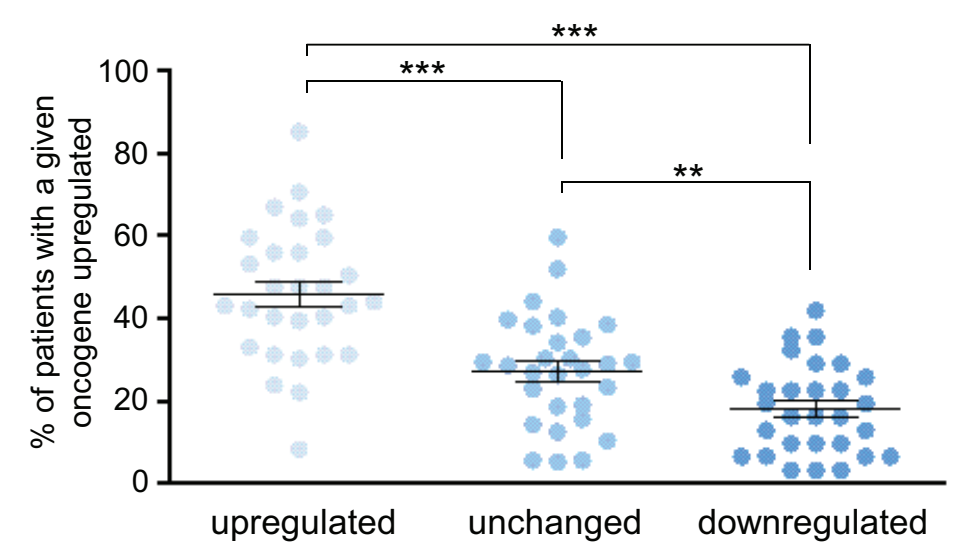

MET levels in HCC patients
C

Predicted Tumour Suppressors

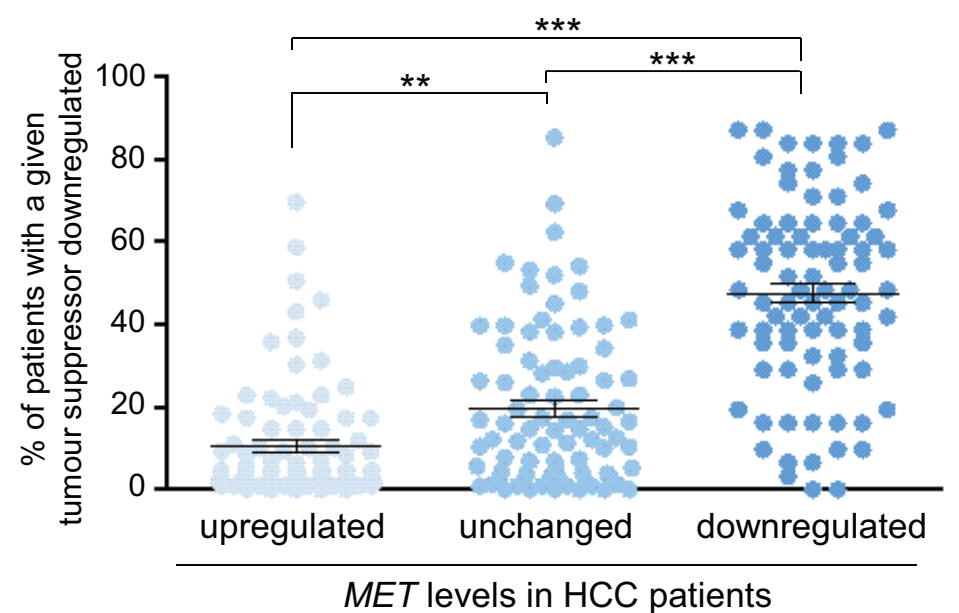




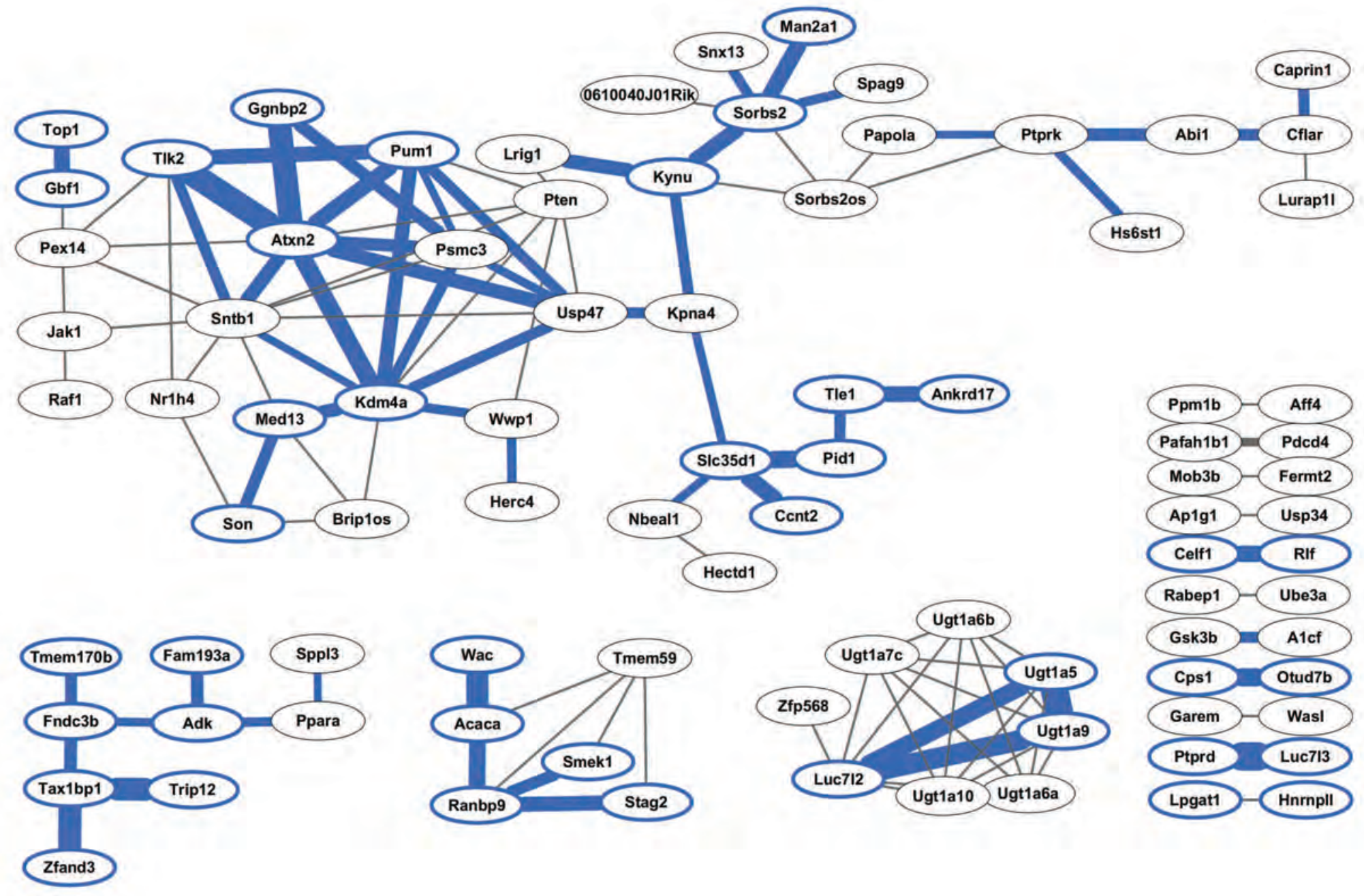

Figure 4 


\section{FAquré5.ll Signalling Pathways}

WikiPathways

Focal Adhesion-PI3K-mTOR sign. Circadian ryhm IL-6 sign.

Androgen Receptor sign. Prot-prot interaction in podocyte TGF $\beta$ sign.

Glycogen metabolism Delta-Notch sign. Notch sign. PluriNetWork Histone modif. IL-7 sign.

TNFa-NFkB sign.

Glycogen metabolism Leptin sign.

mRNA processing

EGF/EGFR sign. TNFa sign.

WNT sign. \& pluripotency Proteasome degradation

\section{Reactome}

Adaptive Immune System Ubiq. \& Proteasome degrad. Develop. Biol EGFR sign.

MHC mediated antigen proces./present. BCR downstream sign. $\beta$-catenin degradation WNT sign. Immune System SCF-KIT sign.

FGFR2 downstream sign. FGFR3 downstream sign. FGFR4 downstream sign. TCF dependent sign. by WNT FGFR1 downstream sign. FGFR4 sign. FGFR3 sign.

Innate Immune System FGFR1 sign.

\section{Kinases}

Kinase Enrichment Analysis

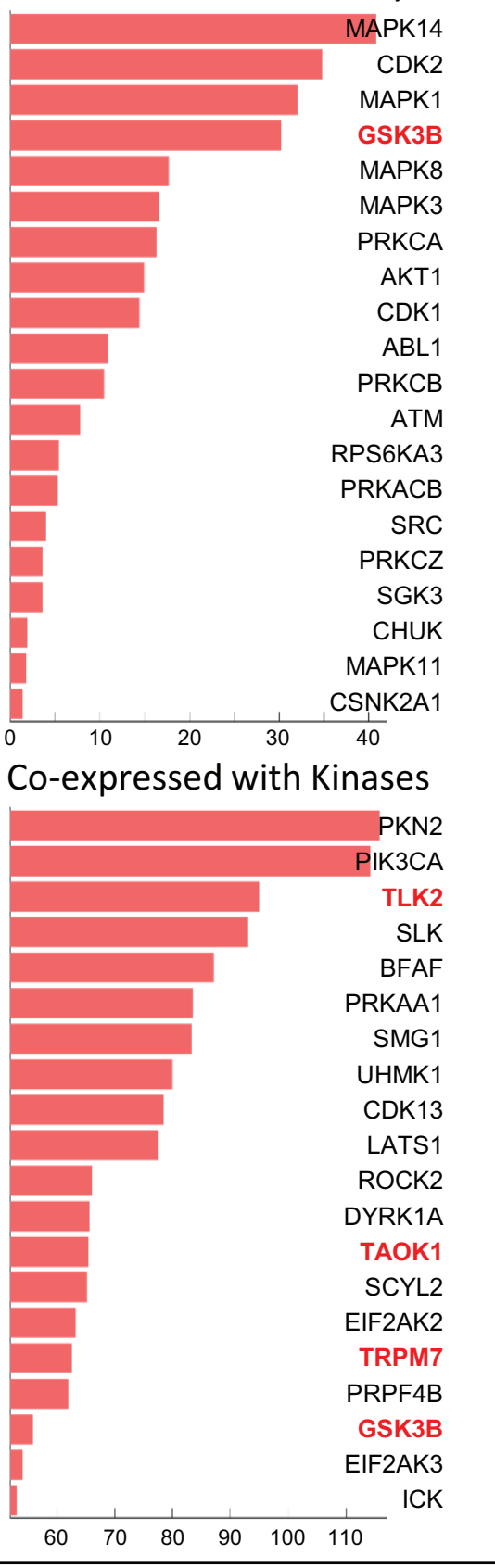

Metabolic pathways from

Kinase Perturbations up

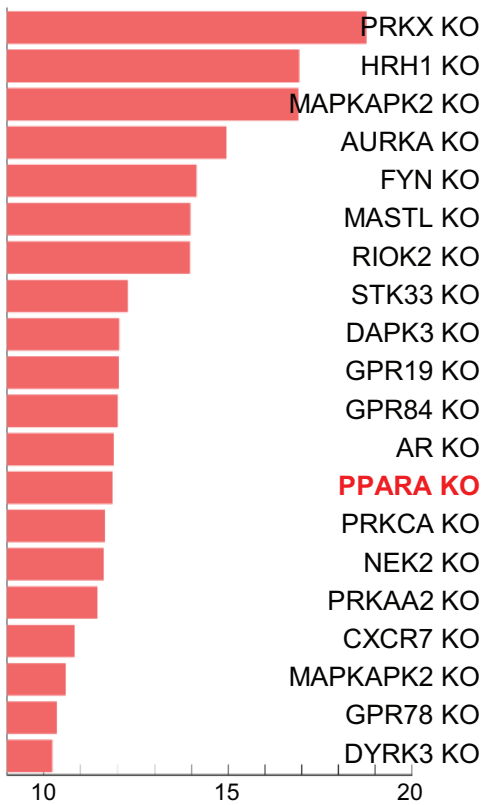

Kinase Perturbations down

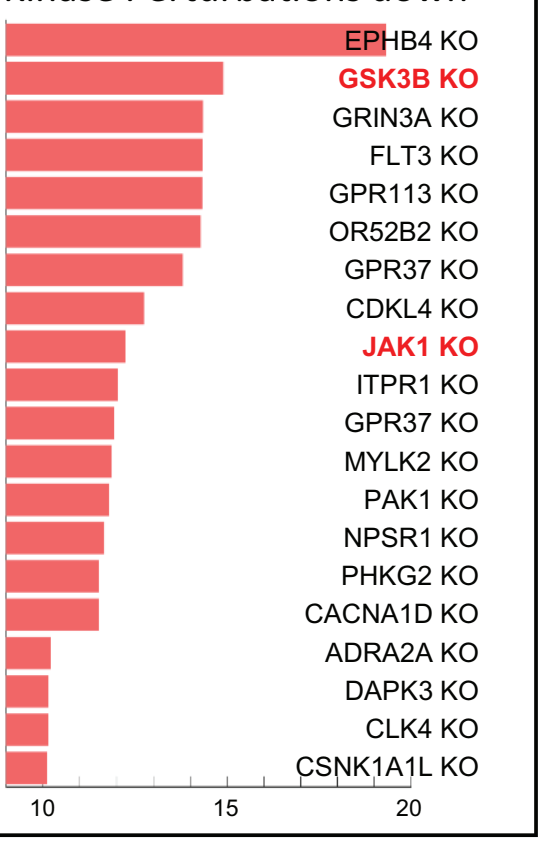

E Human Disease

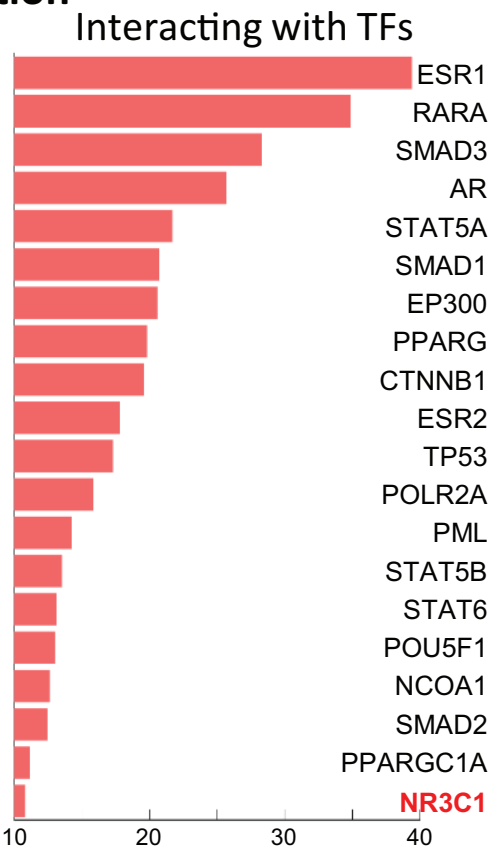

\section{Transcription}

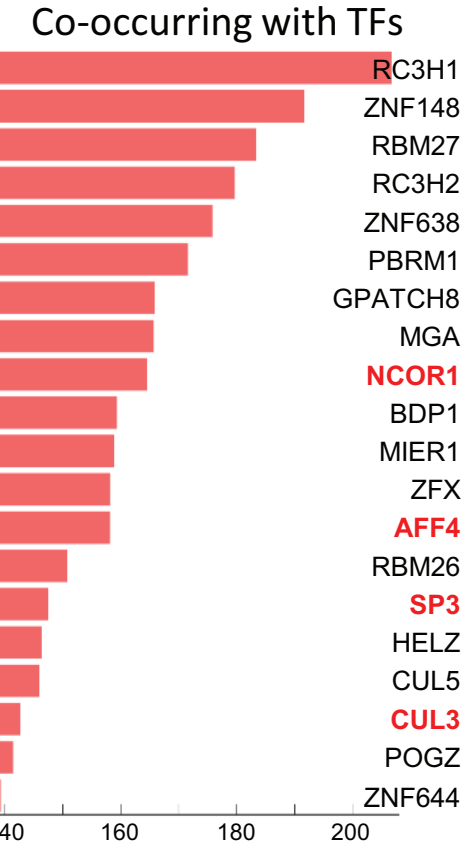

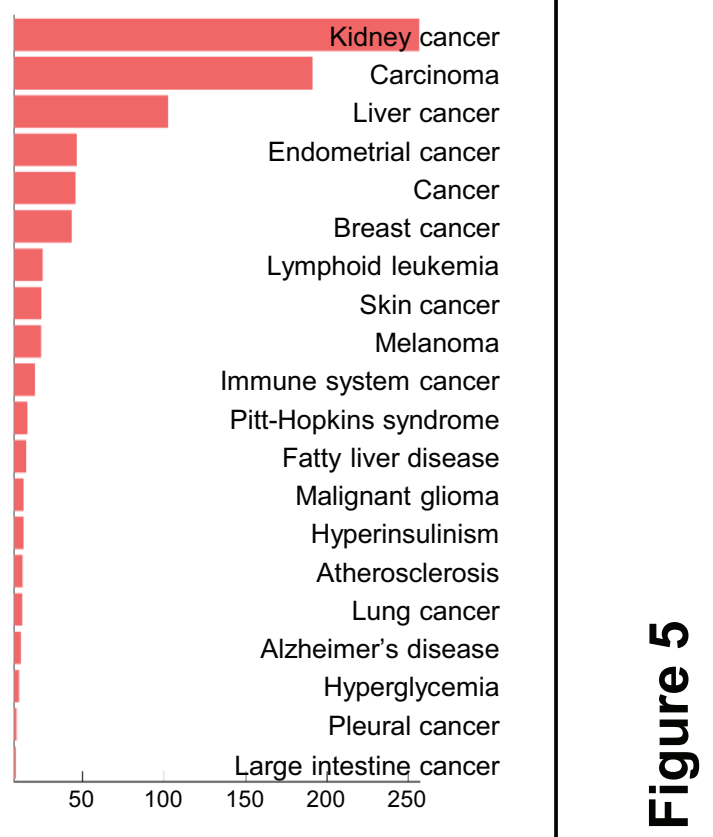


Figure 6

$2^{\text {snxis }}$

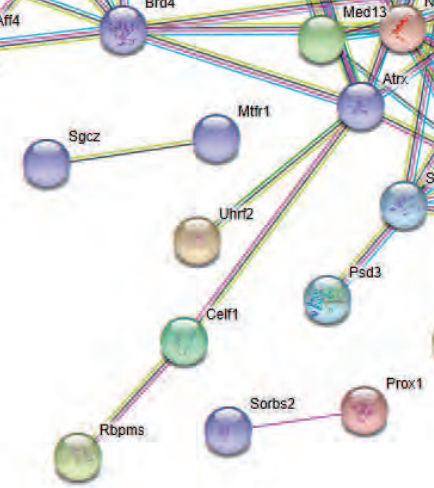

\section{Known interaction}

From curated database

Experimentally determined
Predicted interaction

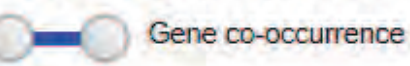

\section{Others}

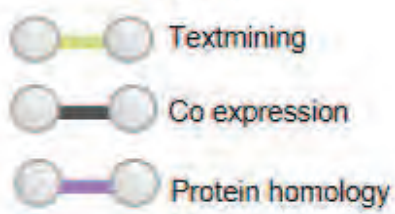

Figure 6 


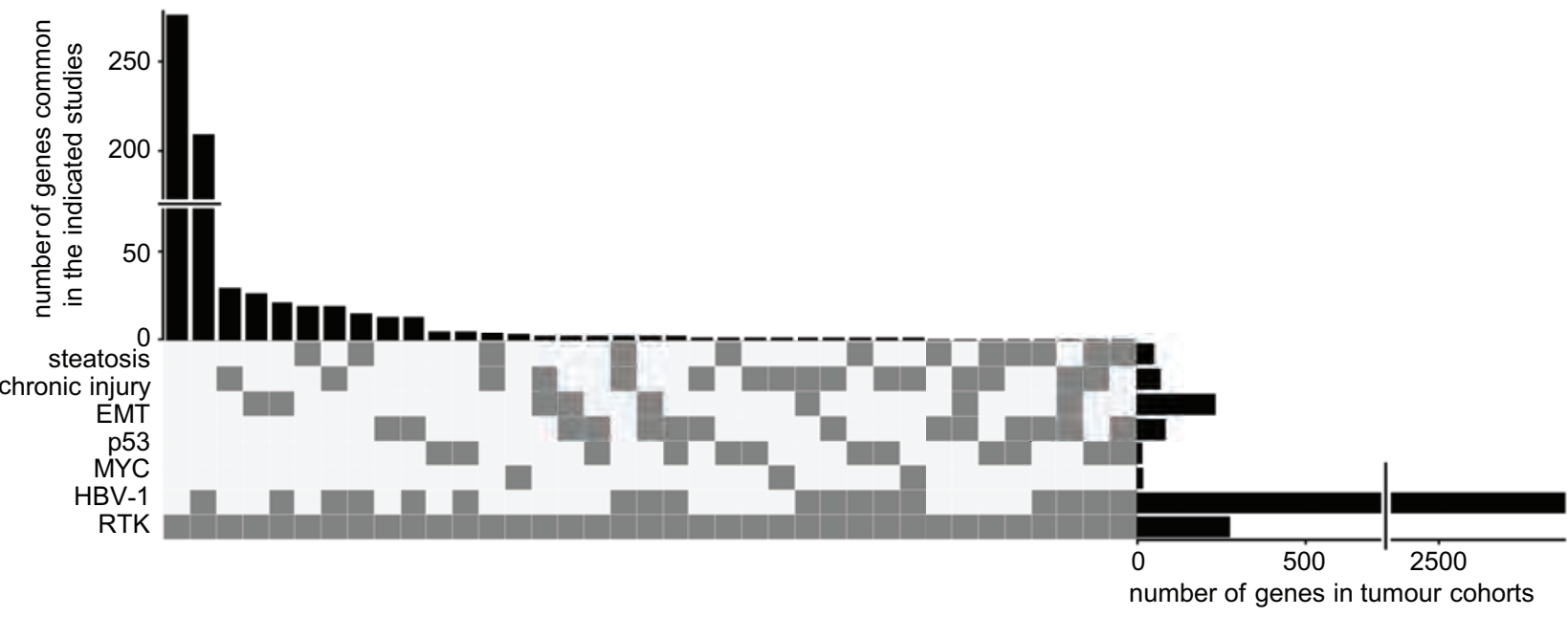

B

number of genes in tumour cohorts

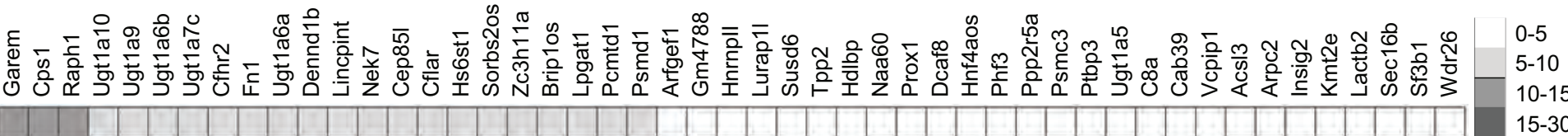
putative "RTK specific" genes

mutation frequency $(\%)$

C

Co-expressed with kinases

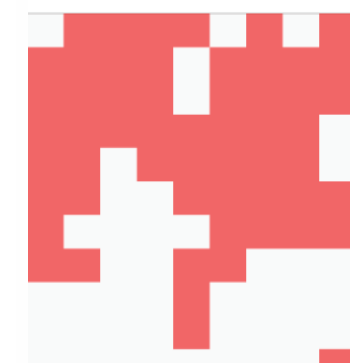

ZC3H11A WDR26 ARFGEF1

PHF3

VCPIP1

CFLAR

SF3B1

CEP85L

DENND1B

PCMTD1

PPP2R5A

CPS1

HDLBP

NEK7

PTBP3

CAB39

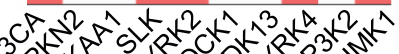

evt?

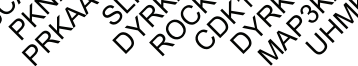

\section{Co-expressed with TFs}

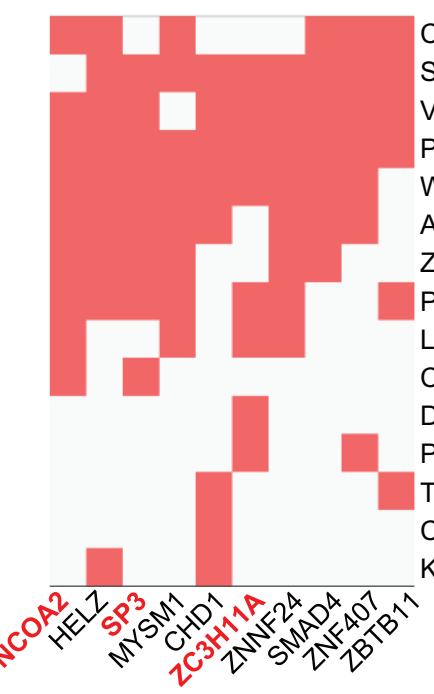

\section{Transcription}

Consensus target genes for TFs

CFLAR SF3B1 VCPIP1 $\mathrm{PHF} 3$

WDR26 ARFGEF1 ZC3H11A PTBP3 LPGAT1 CAB39

DCAF8

PCMTD1

TPP2

CAP85L KMT2E

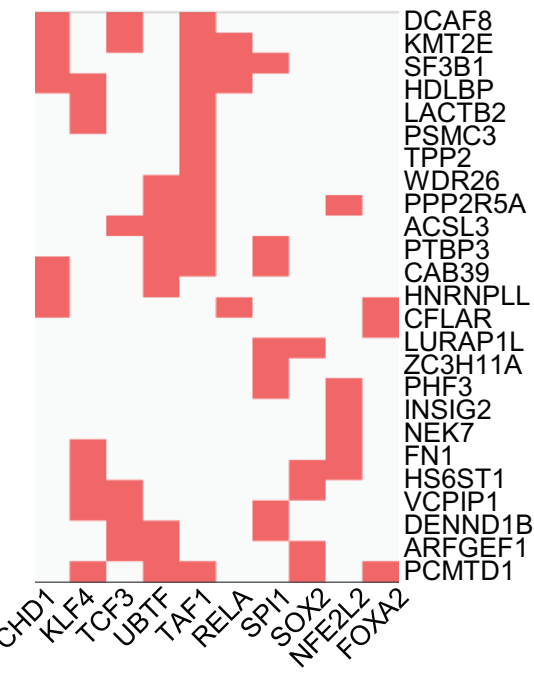

Metabolic pathways from
Kinase Perturbatios up

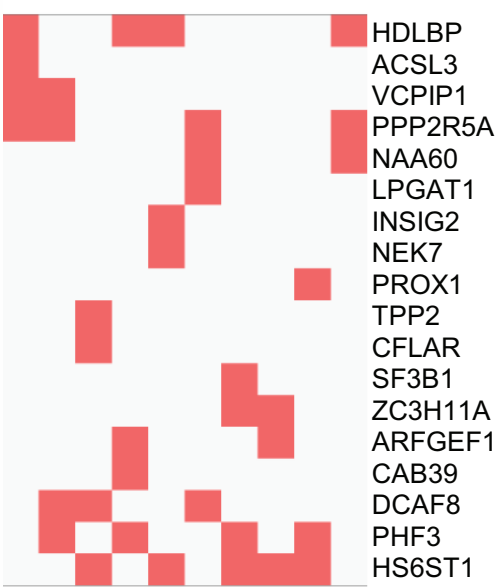

O

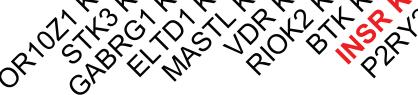

Kinase Perturbation down

PSMC3

PPP2R5A

DENND1B

INSIG2

PHF3

CFLAR

ARPC2

PSMD1

CAB39

HS6ST1

C8A

HDLBP

DCAF8

CPS1

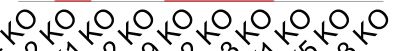
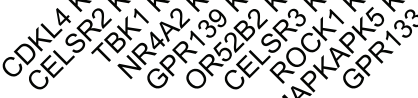

\section{F Biological processes}

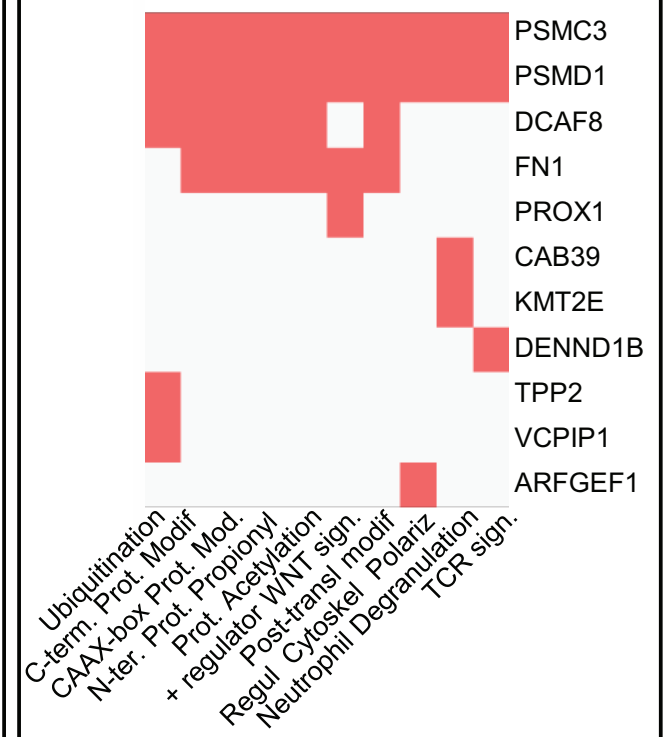


A
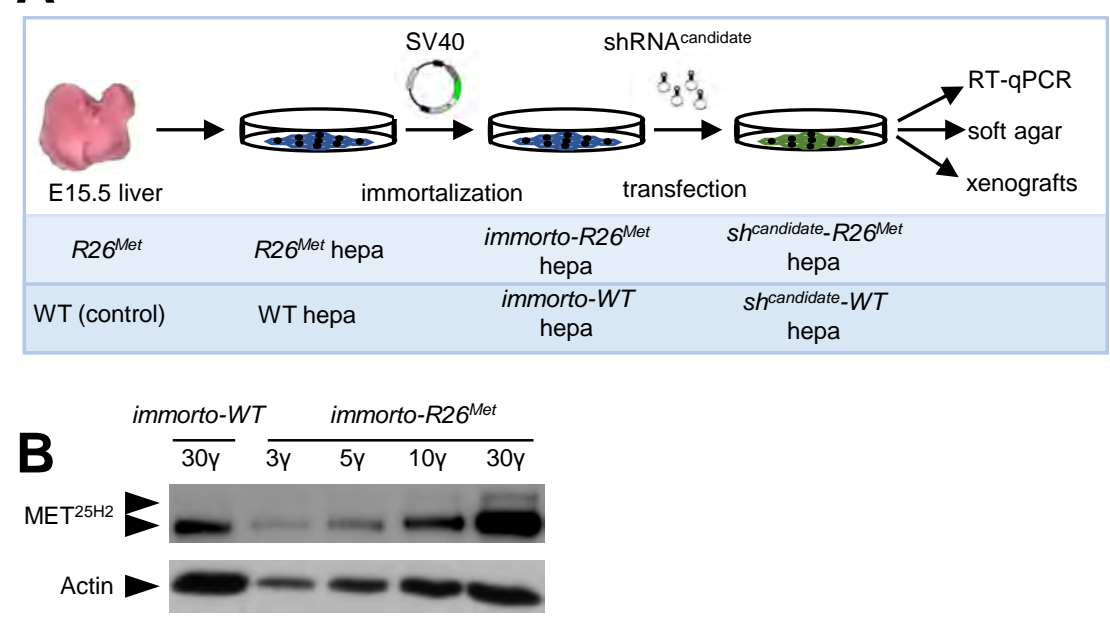

D

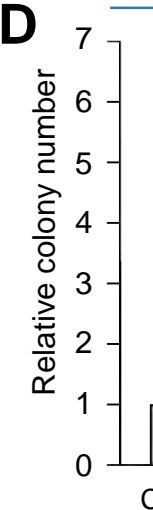

Control Adk Cacul1 Ddi2 Ddx6 Kansl1 Kmt2e Lrch3 Ncor1 Ppp6r3 Ptprd Sorbs2 Stag2 Usp34 Usp47 Wbp1I Zfand3 shRNA targeting sequence

E
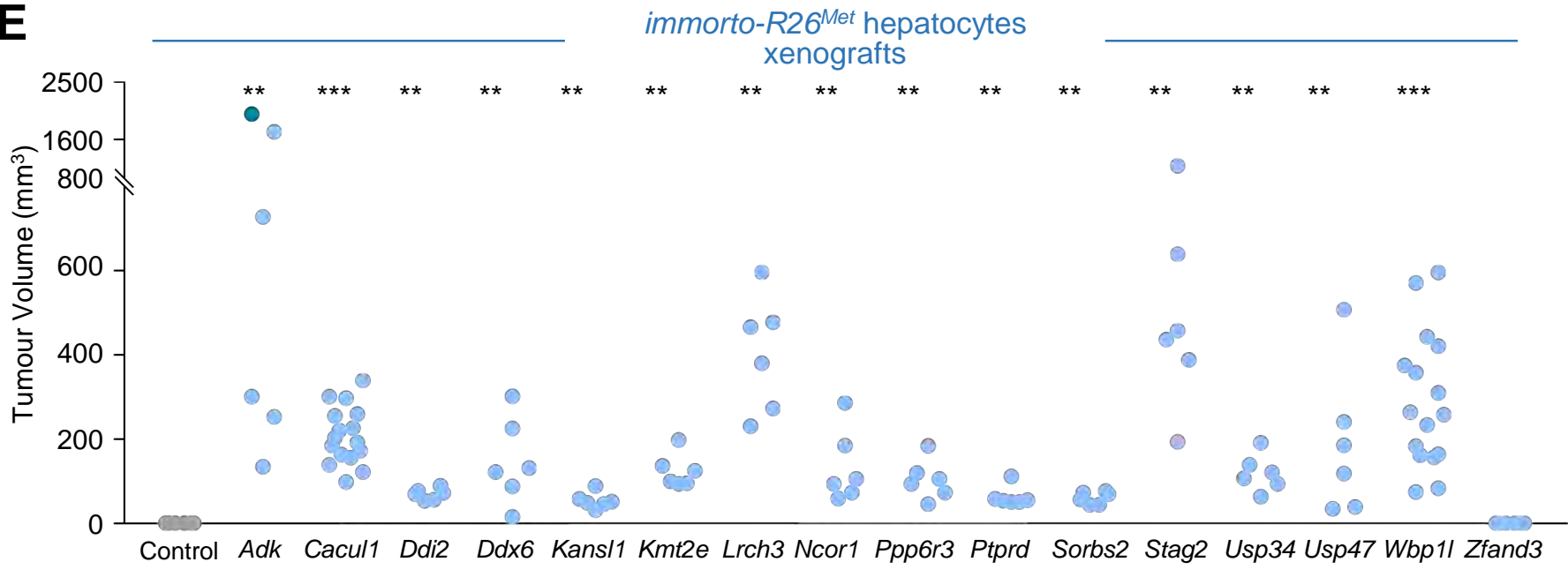

F
C

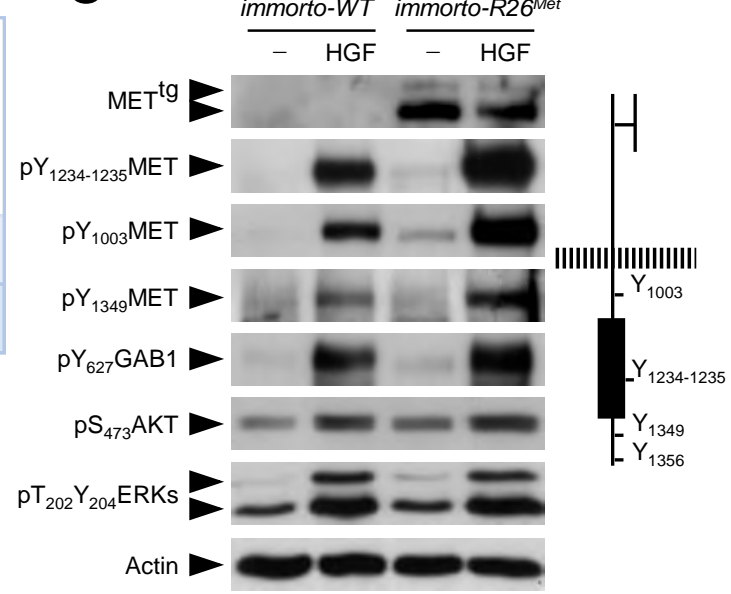

immorto-R26 $6^{\text {Met }}$ hepatocytes anchorage-independent growth assays

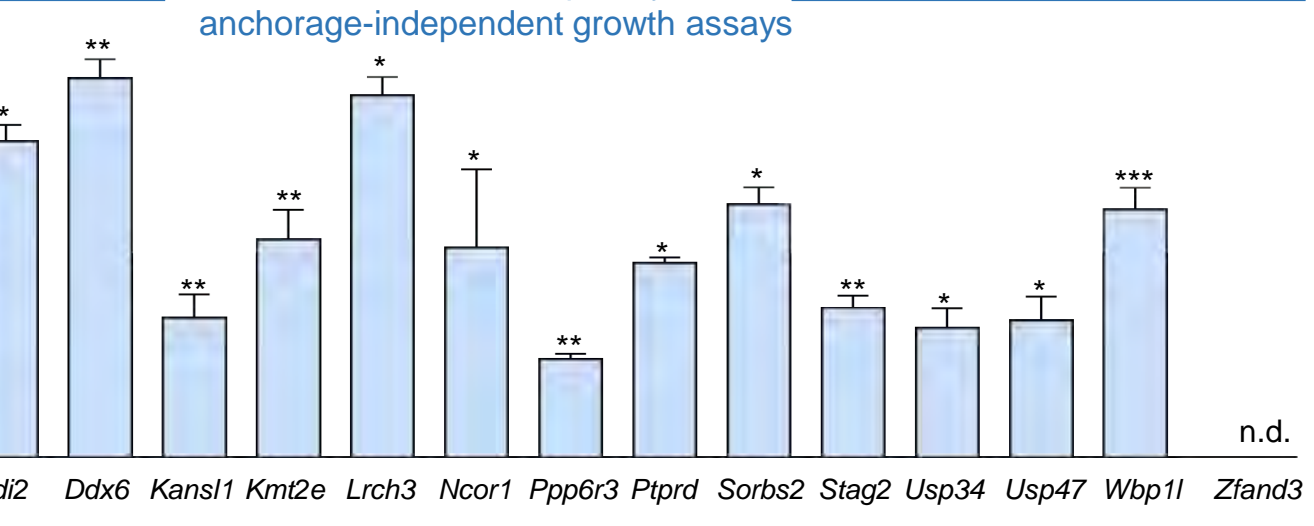

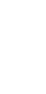




\section{Highlights}

- A transposon genetic screen uncovered 275 putative RTK cooperators for liver cancer

- Most identified genes are also altered in HCC patients

- $\quad$ RTK cooperators are regulators of a large spectrum of cellular functions

- Enhanced RTK levels allow a broad range of mechanisms to initiate liver cancer 